\title{
12. CORRELATION AMONG THE CHANGES WITH ALTERATION IN MINERALOGICAL, CHEMICAL, AND MAGNETIC PROPERTIES OF UPPER OCEAN CRUST, HOLE 896 ${ }^{1}$
}

\author{
J. Honnorez, ${ }^{2}$ B.M. Honnorez-Guerstein, ${ }^{3}$ H.-U. Worm,${ }^{4}$ and C. Laverne ${ }^{5}$
}

\begin{abstract}
Hole 896A was drilled in a 6.83-m.y.-old crust located $1 \mathrm{~km}$ away from Hole 504B, that is, about $200 \mathrm{~km}$ south of the Costa Rica Ridge, in the equatorial east Pacific. It penetrated $290 \mathrm{~m}$ into basement formed by variably altered pillow basalts, with frequent pillow breccias and hyaloclastites, and a few massive lava flows. The average recovery was $28 \%$. Thirty-five samples were collected for magnetic properties measurements, petrographic and mineralogical observations, and bulk-rock chemical analyses. The study shows that all but two of the study samples have been altered by seawater at low temperature, that is, probably at less than $50^{\circ} \mathrm{C}$, as indicated by the rock Curie temperatures averaging $326^{\circ} \mathrm{C}$, high $\mathrm{K}_{2} \mathrm{O}$ contents up to $0.26 \%$, and high Fe oxidation ratio ranging from 0.33 to 0.65 . The samples contain homogeneous Ti-maghemite as the only ferrimagnetic mineral with a size that ranges from 0.5 to $300 \mu \mathrm{m}$, but is generally comprised between 1 and $<10 \mu \mathrm{m}$. In such rocks olivine is often completely replaced by saponite with or without iddingsite and rare celadonitic minerals, whereas plagioclase is replaced by saponite in the most altered samples containing saponite with minor phillipsite veins. Void spaces are filled with saponite, iddingsite, and, more rarely, celadonitic minerals. Calcite is only present in a few samples. Two samples that have holohyaline textures corresponding to a pillow lava rim and a hyaloclastite do not contain ferrimagnetic minerals and are paramagnetic.

Curie temperatures are fairly invariable throughout the hole $\left(T_{r}=326 \pm 31^{\circ} \mathrm{C}\right.$ with a slight increase below 380 mbsf. Saturation magnetization increases downhole, with average values of 0.37 and $0.67 \mathrm{Am}^{2} / \mathrm{kg}$ above and below $330 \mathrm{mbsf}$, respectively. This suggests that the basalts contain almost twice as much ferrimagnetic phases below than above this depth. The saturation magnetization increase with depth is coherent with an increase in magnetic susceptibility and in contrast to a strong decrease in NRM intensity occurring below $364 \mathrm{mbsf}$ (Allerton et al., this volume). The saturation remanence to saturation magnetization ratios of the study samples are $<0.2$ below $330 \mathrm{mbsf}$, whereas they range from 0.2 to 0.45 above this depth, indicating that the upper basalts contain pseudo-single domain grains and, hence, have very stable magnetic properties.

The two unaltered samples are coarse, subophitic basalts with essentially fresh olivine showing only traces of replacement by saponite along cracks and with a relatively large Ti-magnetite content (i.e., 10-200 $\mu \mathrm{m}$ in diameter). These samples have low Curie temperatures $\left(209^{\circ}-250^{\circ} \mathrm{C}\right)$, a low $\mathrm{K}_{2} \mathrm{O}$ content $(0.02 \%)$, and a low oxidation ratio $(0.23)$. They also have low porosities.

Bulk-rock Curie temperatures, $\mathrm{K}_{2} \mathrm{O}$ contents, and oxidation ratio are three parameters whose high values indicate low-temperature alteration of the MORBs by seawater. The presence of titanomaghemite as the sole ferrimagnetic phase in all but two study samples, as confirmed by their small range of Curie temperature values, implies a homogeneous alteration process of the primary titanomagnetite of the basalts. This is in contrast to the extreme variability of the alteration mineralogy and chemistry of the basaltic silicates, which do not show any systematic variation. Therefore, two different low-temperature alteration processes appear to have occurred.
\end{abstract}

\section{INTRODUCTION}

Site 896 is located $1 \mathrm{~km}$ southeast of Hole 504B at a water depth of $3439.8 \mathrm{~m}$. The site was chosen on a basement topographic high that coincides with a heat-flow maximum of $275 \mathrm{~mW} / \mathrm{m}^{2}$. Undetected at such a high heat flow, hydrothermal fluids at moderate temperature are upwelling through the $179-\mathrm{m}$ sediment cover at velocities of a few millimeters per year (Langseth et al., 1988; Mottl, 1989). A temperature of $50^{\circ} \mathrm{C}$ was derived from the measured temperature profile at the sediment/basement interface.

Hole $896 \mathrm{~A}$ is located on a tilted block and at the foot wall of a fault encountered when drilling Hole 504B, which is located on the fault hanging wall of the site (Initial Reports, Vol. 148; see details in

'Alt, J.C., Kinoshita, H., Stokking, L.B., and Michael, P.J. (Eds.), 1996. Proc $O D P$, Sci. Results, 148: College Station, TX (Ocean Drilling Program).

${ }^{2}$ Institut de Géologie and Centre de Géochemie de la Surface, Université Louis Pasteur, I rue Blessig, F-67084 Strasbourg Cedex, France. honnorez@illite.u-strasbg.fr

${ }^{3} 14$, rue du Moulin, F-67206 Mittelhausbergen, France.

${ }^{4}$ Bundesanstalt für Geowissenschaften und Rohstoffe, Stilleweg 2, Postfach510153. D-30631 Hannover. Federal Republic of Germany.

${ }^{5}$ Laboratoire de Pétrologie Magmatique, URA CNRS 1277, Faculté des Sciences et Techniques de Saint-Jérôme, Avenue Escadrille Normandie-Niemen, 13397 Marseille Cedex 20, France. lacte@vmesal1.u-3mrs.fr
Alt, Kinoshita, Stokking, et al., 1993). Hole 896A penetrated $290 \mathrm{~m}$ into a 6.82 -m.y.-old basaltic basement; 30 cores were collected with an average recovery of $28 \%$. The pillow basalts and associated pillow breccias and hyaloclastites comprise $62 \%$ of the drilled section; the remainder is formed by thin massive lava flows and two dikes. The basalts are sparsely to highly phyric tholeiites, generally with plagioclase (the most abundant) and olivine (the second most abundant) phenocrysts. They commonly contain $\mathrm{Cr}$-spinels. Clinopyroxene is present in minor amounts and as partly resorbed phenocrysts from 353.1 to $392.1 \mathrm{~m}$ below seafloor (mbsf). No aphyric basalts were found.

The basalts are extremely depleted tholeiites similar to those from Hole 504B, but the basalts from the uppermost $100 \mathrm{~m}$ of Hole $896 \mathrm{~A}$ are even more depleted than those from Hole 504B. Variations of bulk-rock chemical composition reflect several primary and secondary effects: fractional crystallization and proportions between the various types of phenocrysts, different magmas sources, and posteruptive alteration.

All of the samples recovered from Hole 896A display the effects of low-temperature alteration manifested as secondary phases replacing the basalt primary minerals and/or filling primary voids (such as vesicles, vugs, and miarolitic voids) and secondary spaces (such as veins and breccia cement). Low-temperature alteration is defined 
(Honnorez, 1981) as "ranging from the bottom water temperature up to, but not including, the crystallization temperature of unequivocally metamorphic minerals ..."

The drilled section was divided by the scientific party into upper and lower sections: the magnetic properties (see "Magnetic Properties" section, this chapter) suggest a boundary at 370 mbsf whereas the lithology suggests a boundary at 390.1 mbsf. Finally, there is a marked increase in scatter of the $V_{p}$, and thermal conductivity below $350 \mathrm{mbsf}$.

The purpose of the present work is to study the effect of submarine alteration on seafloor magnetism by correlating the various magnetic properties with mineralogical and chemical composition of basalts from the 290-m-thick section of Hole 896A in the upper oceanic crust.

We will demonstrate that changes with alteration in the bulk properties of ocean-floor basalts depend on the type of alteration, which is always a stepwise process. The final result of low-temperature alteration is a further increase of the upper crust's primary heterogeneity, which is caused by a variety of volcanic emplacement mechanisms.

\section{ANALYTICAL TECHNIQUES Sample Preparation}

Thirty-five samples of basalts were collected, that is, eighteen 19mm-diameter and eleven 24-mm-diameter minicores and six sawedoff slabs with irregular shapes. All of the samples were studied for magnetic properties and in thin sections, but only 29 were chemically analyzed because six samples were too small.

The samples were first measured for magnetic properties; then, a slab was sawed-off from each sample for preparing polished thin sections. Finally, for chemical analysis, the remainder of each sample was crushed, in a steel Abich mortar, and ground up in an agate mill after cleaning its surfaces. When samples exhibited secondary mineral veins, the latter were included in the sample to be crushed and ground up for chemical analyses to be representative of the same mineral assemblage as that measured for magnetic properties. Most samples yielded about 10 to $30 \mathrm{~g}$ of powder. Powder splits of approximately $\mathrm{l} \mathrm{g}$ were used for $\mathrm{Fe}^{2+}$ analyses by titration; the remainder of the powders were used for major element analyses by X-ray fluorescence.

\section{Chemical Analyses}

Major element analyses were performed at the GeoForshungsZentrum Potsdam (GFZ), Germany, using X-ray fluorescence techniques on glass beads fused with lithium metaborate (rock to flux ratio 1:6) using a Siemens SRS 303 AS computerized spectrometer for intensity measurements and a Siemens "alpha" program for concentration calculations; $\mathrm{Fe}^{2+}$ analyses were conducted by manganometric titration; $\mathrm{H}_{2} \mathrm{O}$ and $\mathrm{CO}_{2}$ were determined with a Leco RC 412 analyzer after thermal decomposition of the rock powder at $1100^{\circ} \mathrm{C}$ under oxygen atmosphere (at GFZ). The precision of major element determinations was tested by duplicate measurements of selected samples. Accuracy was checked by running the reference rock standards BM and BM 10 as "unknown" through the whole procedure.

\section{Magnetic Properties Measurements}

Rock magnetic characterizations included Curie temperature $\left(T_{c}\right)$ and hysteresis parameter determinations. The saturation magnetization was measured as a function of temperature for $T_{c}$ determinations in the laboratory Grubenhagen of the BGR, Germany, with a Curie balance operating in vacuum on millimeter-sized samples of about $150 \mathrm{mg}$. The applied field strength was $0.55 \mathrm{~T}$ and a heating rate of
$20 \% \mathrm{~min}$. was employed. The vacuum and the rather large grain size reduce oxidation during heating that would result in higher $T_{c}$ values.

Hysteresis properties, coercivity $\left(H_{c}\right)$, and saturation remanence over saturation magnetization $\left(M_{r s} / M_{s}\right)$ have been determined with a vibrating sample magnetometer at the Institute for Rock Magnetism, University of Minnesota, Minneapolis, MN, U.S.A. Peak fields were 1 Tesla. Magnetic properties were measured before grinding.

\section{PETROGRAPHY AND MINERALOGY}

Thirty-five polished thin sections were studied in transmitted and reflected lights: textures and structures were observed and primary and secondary phases were identified on the basis of their optical properties. Petrographical and mineralogical observations are summarized in Table 1.

\section{Colors and Textures}

In hand specimen, the samples are gray where least altered and display various shades of brown where altered. This discoloration is commonly related to the vicinity of open spaces such as cracks, vugs, or miarolitic voids. Reddish brown halos are generally adjacent to exposed surfaces such as pillow rims or fissures. The brown color in thin sections is caused by unspecified Fe-oxyhydroxides, goethite, or iddingsite, that is, mixtures between smectite and Fe-oxyhydroxide replacing olivine phenocrysts, staining plagioclase crystals, of sheaf or plumose mesostasis, or filling voids. Remarkably palagonitized glasses do not exhibit brownish colors but are very light colored in thin section.

Sample texture varies from holohyaline to intergranular, with all the transitional textures indicating progressive crystallinity from the lava surface exposed to quenching by seawater, as in glassy pillow rims, to discrete and coalesced varioles and honeycomb textures of the variolitic zone, to plumose, to sheaf, to branching or comb textures of the subvariolitic zone. We followed the descriptive nomenclature used in the "Igneous Petrology" section of Chapter 3 (Alt, Kinoshita, Stokking, et al., 1993).

We assume that the ever-present and abundant dark groundmass displaying plumose, sheaf, or comb textures are essentially made up of clinopyroxene. Therefore, the latter mineral will not be mentioned in the summary description of the mesostasis in Table 1. Similarly, we do not mention in Table 1 the plagioclase microlites, which are almost always very abundant in the groundmass, except in samples with variolitic textures. On the other hand, the characteristics of the primary Fe-Ti oxides are reported in Table 1 because the opaque minerals are the ferrimagnetic phases responsible for the sample magnetic properties.

\section{Primary Mineralogy}

Plagioclase phenocrysts and microlites are generally completely unaltered (see Pls. 1, 3) except when they are located near larger smectite veins (see Pl. 2), such as in Samples 148-896A-21R-2, 117 $119 \mathrm{~cm}$ (Piece 11B), 23R-2, 19-21 cm (Piece 2A), 25R-1, 140-143 $\mathrm{cm}$ (Piece 17B), and 26R-2, 117-120 cm (Piece 18B), and/or in areas containing numerous vugs filled with smectite, such as in Samples 148-896A-9R-1, 31-33 cm (Piece 6), 25R-1, 140-143 cm (Piece 17B), and 21R-2, 117-119 cm (Piece 11B). The latter sample, in which plagioclase appears to be most intensively replaced by smectite, contains both veins and vugs filled with smectite. When altered, the plagioclase phenocrysts commonly exhibit an unaltered rim of albitic composition, that is, so-called "gefüllt plagioclase" (meaning "stuffed plagioclase"; Heinrich, 1965) (see Pl. 2, Figs. 1, 2, 3).

Olivine phenocrysts are almost always completely replaced by coarsely crystallized smectite with or without iddingsite and/or Fe- 
oxyhydroxides (see Pl. 1, Fig. 2, and Pl. 2, Fig. 1, for example with iddingsite). Olivine phenocryst alteration can result in a box-work texture in which ribbons of Fe-oxyhydroxides occasionally identified as goethite separate smectitic areas (see Pl. 3, Fig. 3).

Olivine relicts were found in three samples (see Table 1) and remained essentially fresh in only one sample (148-896A-6R-3, 107$110 \mathrm{~cm}$, Piece 21; see PI. 1, Fig. 1), even though the sample has a $0.01-\mathrm{mm}$-thick smectite vein and numerous vugs filled with smectite.

Discrete clinopyroxene and spinel crystals are all unaltered.

In reflected light the opaque minerals are light brown in color and isotropic, and they always appear to be homogeneous irrespective of their grain size. We could not ascertain whether they are actually titanomagnetite or titanomaghemite because no single grain of opaque mineral displays, side by side, the contrasted optical properties of both these phases. Therefore, in the following pages, opaque minerals will be called "Fe-Ti oxides."

$\mathrm{Fe}-\mathrm{Ti}$ oxides occur as very small, irregularly shaped grains and/or skeletal crystals, which generally are less than $5 \mu \mathrm{m}$ in diameter (see PI. 4, Figs. 1, 2). Samples 148-896A-11R-3, 89-91 cm (Piece 15), 16R-2, 26-28 cm (Piece IC), and 16R-3, 84-86 cm (Piece 4), contain exceptionally large skeletal crystals, up to $300 \mu \mathrm{m}$ long (see PI. 5, Figs. 1,2). In these three samples with exceptionally large opaque crystals, the Fe-Ti oxide appears to be unoxidized (see "Discussion" section, this chapter).

Drop-shaped sulfide grains were found as tiny, discrete grains $(<2$ $\mu \mathrm{m}$ in diameter) scattered through the groundmass, or attached to the Fe-Ti oxides in Samples 148-896A-11R-3, 89-91 cm (Piece 15), and 16R-3, 84-86 cm (Piece 4) (see Pl. 5, Fig. 2). Some of the dropshaped sulfides were observed to be replaced by goethite.

\section{Secondary Mineralogy}

All 35 study samples contain secondary phases indicating lowtemperature alteration by seawater (see Tables 1,2). One must distinguish between diffuse background alteration affecting the whole of the rock from brown alteration halos and bands restricted to areas typically adjacent to fractures and other exposed surfaces.

Secondary minerals are present either as replacement products of primary basaltic phases or as fillings of the primary porosity. The latter is formed by open cracks, miarolitic voids, and vugs presently filled by secondary phases, that is, generally saponite and iddingsite, and more rarely celadonitic minerals, phillipsite, or unidentified Feoxyhydroxides. The secondary minerals generally precipitated as successive layers with the Fe-rich phases nearest to the void walls (see Pl. 3, Figs. 1, 2). Interstitial glass or cryptocrystalline areas of the groundmass differ in replacement by homogeneous extremely fine saponite.

Secondary minerals were identified by their optical properties in thin-sections, by X-ray diffraction and electron microprobe analysis (for analytical conditions, see Laverne et al., this volume). All of the smectitic clay minerals from Hole 896A studied by X-ray diffraction and electron microprobe are saponites.

Saponite (saponite types 1 and 2 of Laverne et al., this volume) is by far the most abundant secondary mineral. It displays three contrasting habits according to its occurrence:

1. Homogeneous, light tan to pale brown or more rarely reddish, very finely crystallized saponite replaces interstitial glass in the groundmass (PI. 1, Fig. 2; Pl. 3, Fig. 3). It exhibits very low birefringence.

2. Tan or variously colored, coarsely crystallized saponite forms with (Pl. 1, Fig. 2; Pl. 2, Fig. 1) or without (Pl. 1, Fig. 2; Pl. 2, Fig. 3) iddingsite and Fe-oxyhydroxide olivine pseudomorphs. This type of saponite generally exhibits low to medium birefringence, up to the second order green.

3. Colorless to $\tan$ to brown fibrous saponite replaces plagioclase (Pl. 2, Figs. 1, 2, 3) or fills cracks (Pl. 1, Fig. 3; Pl. 2, Figs. 1,
2, 3) and vugs (Pl. 3, Figs. 1, 2). It displays low birefringence, up to the first-order yellow.

When associated with iddingsite or Fe-oxyhydroxides, saponite occupies the central portion of the voids, whereas the Fe-rich phases is near the void walls (Pl. 1, Fig. 2; PI. 2, Fig. 1; Pl. 3, Figs. 1, 2).

Iddingsite and Fe-oxyhydroxides (types $8 \mathrm{a}$ and $8 \mathrm{~b}$ of Laverne et al., this volume) are generally associated with saponite in olivine pseudomorphs (PI. 1, Fig. 2; PI. 2, Fig. 1) and in vein and vug fillings (Pl. 3). The secondary Fe-rich phases, some of which were identified as goethite, typically located at the edges of or along fissures within olivine phenocrysts. Plagioclase phenocrysts and groundmass plumose and sheaf-like textures are stained in reddish or orange-brown colors along saponite veins or around saponite-filled vugs generating the macroscopic reddish or brownish halos.

Celadonitic minerals, that is, a mixture of green, $\mathrm{K}$ - and Fe-rich clay minerals were observed in eight of the study samples. Three types of celadonitic minerals were tentatively distinguished on the basis of their optical properties and chemical compositions (Laverne et al., this volume): celadonite (type 3 ), celadonite-saponite mixtures (type 4), and celadonite-nontronite mixtures (type 5). The samples that contain the largest amounts of green clay minerals are 148-896A17R-2, 18-20 cm (Piece 3A), and 30R-1, 97-99 cm (Piece 12B). In both samples, celadonitic minerals occur with saponite as a replacement for plagioclase phenocrysts and filling cracks, vugs, and miarolitic voids. In the latter three cases, celadonitic minerals are usually located near the void walls whereas saponite occupies the central part. In the six other samples, the green clay mineral generally occurs with saponite either as a plagioclase replacement or as a void filling. Iddingsite is commonly associated with celadonitic minerals.

Phillipsite was observed forming clusters of small prismatic crystals imbedded in saponite-filled veins in Samples 148-896A-17R-2, 18-20 cm (Piece 3A), and 21R-2, 117-119 cm (Piece 11B) (Pl. 2, Fig. 3). A few rare crystals of an unidentified zeolite were observed near the center of a smectite vein with iddingsitic selvage in Sample 148-896A-25R-1, 140-143 cm (Piece 17R).

Calcium carbonates were exceptionally observed but are never abundant in four samples. Calcite is present as blocky crystals in the center of a partially lost vein with one saponite selvage preserved at one side of Sample 148-896A-1R-1, 47-55 cm (Piece 11). A fibrous calcium carbonate, tentatively identified as aragonite, is also present at the center of an iddingsite-rich veinlet crossing earlier Fe-oxyhydroxides and saponite veinlets, and associated with smectite in miarolitic void fillings in Sample 148-896-3R-1, 83-86 cm (Piece 9). Finally, a calcium carbonate is associated with saponite and iddingsite and, in a few rare cases, with olivine pseudomorphs in Samples 148-896A16R-3, 84-86 cm (Piece 4), and 17R-1, 136-137 cm (Piece 16A).

Pyrite and Marcasite are present in saponite veins in two samples (148-896A-6R-3, 107-110 cm, Piece 21, and 24R-5, 117-119 cm, Piece 12 , respectively).

\section{CHEMISTRY}

Bulk-rock chemical analyses of 32 of the 35 study samples are presented in Table 3. One is tempted to explain the chemical composition variations caused by alteration in terms of the observed secondary mineralogy. In the present case, this approach did not work very well. For instance, no relationship exists between the occurrence of calcium carbonates observed in thin sections of four samples and the $\mathrm{CO}_{2}$ content of these samples. The four samples have a $\mathrm{CO}_{2}$ content that falls within the $0.15-0.40$ range of all the analyzed samples. Conversely, the five chemical analyses with exceptionally high $\mathrm{CO}_{2}$ values ranging from $0.65 \%$ to $1.25 \%$ correspond to samples for which the sections did not appear to contain any calcium carbonate. This does not imply that no calcium carbonate existed in the sample portions that were chemically analyzed. Instead, the lack of relationship 
Table 1. Summary of petrographic and mineralogical descriptions.

\begin{tabular}{|c|c|c|c|c|c|c|c|c|c|c|c|c|}
\hline \multirow{3}{*}{$\begin{array}{l}\text { Core, section, } \\
\text { interval }(\mathrm{cm})\end{array}$} & \multirow[b]{3}{*}{ Piece no. } & \multirow[b]{3}{*}{ Color } & \multirow[b]{3}{*}{ Texture } & \multicolumn{4}{|c|}{ Phenocrysts and discrete crystals } & \multicolumn{5}{|c|}{ Mesostasis } \\
\hline & & & & \multirow[b]{2}{*}{ PI } & \multirow[b]{2}{*}{ OI } & \multirow[b]{2}{*}{ Sp } & \multirow[b]{2}{*}{ Cpx } & \multicolumn{2}{|c|}{ Fe-Ti oxides } & \multirow[b]{2}{*}{ Sulfide } & \multirow[b]{2}{*}{$\mathrm{Fe}(\mathrm{OH})_{\mathrm{n}}$} & \multirow[b]{2}{*}{ Glass } \\
\hline & & & & & & & & Abundance & Size $(\mu \mathrm{m})$ & & & \\
\hline \multicolumn{13}{|l|}{ 148-896A- } \\
\hline IR-1,47-55 & 11 & $\mathrm{Br}^{\mathrm{a}}$ & Branching & ++ & $+\rightarrow \mathrm{Sa}$ & - & & - & $<5$ & & & \\
\hline $\begin{array}{l}2 R-1,0-5 \\
3 R-1.83-86\end{array}$ & $\begin{array}{l}1 \\
9\end{array}$ & $\mathrm{Br}$. & Fine intergr. & t+ & $\begin{array}{l}+\rightarrow \text { Sa } \\
+\rightarrow I d\end{array}$ & - & - & $\bar{t}$ & $<5$ & & $\overline{-}$ & $\rightarrow \mathrm{Sa}$ \\
\hline & & & Fine intergr. & + & $\overrightarrow{\mathrm{Sa}}$ & & & & & & & \\
\hline $5 R-2,26-29$ & IC & Gr. & & ++ & $-\rightarrow \mathrm{Sa}$ & - & & - & $<5$ & & & \\
\hline $5 R-2,124-126$ & 13 & Gr. & Plumose-sheaf & + & $\begin{array}{l}+\rightarrow \text { Sa } \\
\text { Relicts }\end{array}$ & -- & & -- & $<5$ & - & & \\
\hline $6 \mathrm{R}-1,21-23$ & 2 & Gr. & Sheaf-branching & ++ & $+\rightarrow \mathrm{Sa}$. Id & - & & - & $<5$ & & - & $\rightarrow \mathrm{Sa}$ \\
\hline $6 \mathrm{R}-1,47-49$ & $5 \mathrm{~B}$ & Mott. gr. & Sheaf-branching-comb. & ++ & $\begin{array}{l}+\rightarrow \text { Sa } \\
++ \text { Relicts }\end{array}$ & -- & + & - & $<5$ & & - & \\
\hline $6 \mathrm{R}-2,67-70$ & 8 & Gr. & Sheaf-branching & ++ & $+\rightarrow \mathrm{Sa}$ & -- & & -- & $<5$ & & & \\
\hline $6 R-3,107-110$ & 21 & & Sheaf-branching-intergr. ${ }^{\mathrm{c}}$ & ++ & + Fresh & - & & -- & $<2$ & & & \\
\hline $7 \mathrm{R}-1,9-15$ & 3 & Brsh. gr. & Coalesced varioles-plumose & $=\quad++$ & $+\rightarrow \mathrm{Sa}$ & - & & & & $\begin{array}{l}\text { Py in } \\
\text { vein }\end{array}$ & & Palagonite \\
\hline $8 \mathrm{R}-1,92-98$ & 19 & Brsh. gr. & Plumose-sheaf & +t & $\rightarrow$ Sa & & & - & & & & Palagonite \\
\hline $\begin{array}{l}9 \mathrm{R}-1,4-8 \\
9 \mathrm{R}-1,31-33\end{array}$ & $\begin{array}{l}2 \\
6\end{array}$ & $\begin{array}{l}\text { Gr. } \\
\text { Mott.gr. }\end{array}$ & $\begin{array}{l}\text { Discrete varioles-plumose } \\
\text { Comb.-fine intergr. }\end{array}$ & $\stackrel{+}{+} \mathrm{Sa}$ & $+\rightarrow \mathrm{Sa}$ & - & & - & $<5$ & $\ldots$ & & Palagonite \\
\hline $10 R-1,79-82$ & $8 \mathrm{C}$ & Mott. gr. & Sheaf-comb.-intergr. & ++ & $+\rightarrow \mathrm{Sa}$ & - & -- & + & $<5$ & - & - & \\
\hline $11 \mathrm{R}-1,50-52$ & $3 \mathrm{E}$ & Mott. gr. & Comb.-fine intergr. & ++ & $\rightarrow \mathrm{Sa}$ & $\ldots$ & -- & + & $<5$ & & & \\
\hline $11 R-3,89-91$ & 15 & Gr. & Coarse intergr. & ++ & $\begin{array}{c}++\rightarrow \text { Sa } \\
\text { Relict }\end{array}$ & - & + & + & $10-50$ & $t^{\mathrm{e}}$ & & \\
\hline $\begin{array}{l}12 \mathrm{R}-1,52-54 \\
15 \mathrm{R}-1,127-129\end{array}$ & $\begin{array}{r}8 \\
18\end{array}$ & $\begin{array}{l}\text { Mott. gr. } \\
\text { Gr. }\end{array}$ & $\begin{array}{l}\text { Comb.-fine intergr. } \\
\text { Plumose-sheaf }\end{array}$ & $\begin{array}{l}++ \\
++\end{array}$ & $\begin{array}{l}+\rightarrow \text { Id, Sa } \\
+\rightarrow S_{3}\end{array}$ & - & & $\stackrel{+}{-}$ & $<2$ & & & \\
\hline $16 \mathrm{R}-2,26-28$ & IC & Grsh. br. & Coarse intergr. & $\begin{array}{l}++ \\
++\end{array}$ & $+\rightarrow \mathrm{Sa}$ & - & + & + & $50-150$ & & + & \\
\hline $16 \mathrm{R}-3,84-86$ & 4 & Grsh. br. & Coarse intergr. & ++ & $+\underset{I d}{+}+\stackrel{l d}{\rightarrow}$ Sa & - & + & + & $50-300$ & & - & \\
\hline $17 \mathrm{R}-1,136-137$ & $16 \mathrm{~A}$ & Gr. & Plumose-comb. & ++ & $+\underset{\mathrm{Ca}^{\mathrm{h}}}{\rightarrow} \mathrm{Sa}$ & -- & + & - & $<5$ & - & & \\
\hline $17 \mathrm{R}-2,18-20$ & $3 \mathrm{~A}$ & Gr. & Comb.-fine intergr. & ++ & $\begin{array}{c}++\rightarrow \text { Sa, Id, } \\
\text { Go, C-N }\end{array}$ & & + & - & $<5$ & & + & \\
\hline $18 \mathrm{R}-1,126-128$ & $11 \mathrm{~B}$ & Gr. & Plumose-comb. & ++ & $\begin{array}{l}+\rightarrow \rightarrow \mathrm{Sa} \\
(\mathrm{C}-\mathrm{N})\end{array}$ & & & - & $<2$ & - & & \\
\hline $21 R-2,117-119$ & $11 \mathrm{~B}$ & Brsh. gr. & Comb.-fine intergr. & $+\rightarrow \mathrm{Sa}$ & $+\rightarrow \mathrm{Sa}$ & & & - & $1-5$ & - & & \\
\hline $22 R-4,8-10$ & IB & Gr. & Branching-fine intergr. & $++\rightarrow \mathrm{Sa}$ & $++\rightarrow$ Id, $\mathrm{Sa}$ & & & + & $2-10$ & & - & \\
\hline $23 R-2,19-21$ & $2 \mathrm{~A}$ & $\mathrm{Br}$. & Comb.-fine intergr. & $++\rightarrow \mathrm{Sa}$ & $\begin{array}{l}+\rightarrow \text { Sa, } \\
\text { Id, Go }\end{array}$ & - & + & - & $2-20$ & - & + & \\
\hline $24 \mathrm{R}-1,15-18$ & 1 & Gr. & Coarse suboph. & ++ & $\begin{array}{c}+\rightarrow \text { C-N, } \\
\text { Relicts } \\
\text { Res }\end{array}$ & - & ++ & + & $10-200$ & + & & $\rightarrow \mathrm{Sa}$ \\
\hline $24 \mathrm{R}-1,12 \mathrm{I}-123$ & 8 & Gr. & Coarse suboph. & ++ & $\begin{array}{l}++\overrightarrow{+} \mathrm{Sa}, \\
\text { Id, C-N, } \\
\text { Relicts }\end{array}$ & - & ++ & + & $10-200$ & + & & $\rightarrow \mathrm{Sa}$ \\
\hline $24 \mathrm{R}-3,51-53$ & 5 & Br. & Coarse integr. & + & $\begin{array}{l}+\rightarrow \mathrm{Sa} \\
\mathrm{Id}, \mathrm{C}-\mathrm{N}\end{array}$ & - & ++ & + & $2-150$ & & + & $\rightarrow \mathrm{Sa}$ \\
\hline $24 R-5,117-119$ & 12 & Mott. br. & Medium integr. & ++ & $\begin{array}{l}+++\overrightarrow{S a} \text {, } \\
\text { Id, C-N. } \\
\text { Relicts }\end{array}$ & & ++ & + & $2-130$ & $\begin{array}{l}\text { Mc in } \\
\text { vein, Py }\end{array}$ & & $\rightarrow \mathrm{Sa}$ \\
\hline $25 \mathrm{R}-1,140-143$ & $17 \mathrm{~B}$ & $\mathrm{Br}$ & Sheaf-branching & $+\rightarrow \mathrm{Sa}$ & $\stackrel{+\rightarrow \mathrm{Sa}}{\mathrm{ld}, \mathrm{Go}}$ & - & & -- & $1-2$ & & & \\
\hline $\begin{array}{l}26 \mathrm{R}-2,117-120 \\
27 \mathrm{R}-3,18-20\end{array}$ & $\begin{array}{r}18 \mathrm{~B} \\
3 \mathrm{~A}\end{array}$ & $\begin{array}{l}\text { Mott, gr. } \\
\text { Gr. }\end{array}$ & $\begin{array}{l}\text { Comb.-fine intergr. } \\
\text { Plumose-sheaf }\end{array}$ & $\begin{array}{l}++\rightarrow \text { Sa } \\
++\rightarrow \text { Sa }\end{array}$ & $\begin{array}{l}+\rightarrow \mathrm{Sa} \\
++\rightarrow \mathrm{Sa}\end{array}$ & - & $1-5$ & -. & $0.5-5$ & & - & \\
\hline $28 \mathrm{R}-1,119-122$ & 18D & $\mathrm{Gr}^{\mathrm{m}}$ & Sheaf & ++ & $+\rightarrow \mathrm{Sa}$ & - & & -- & $0.5-2$ & & & \\
\hline $30 R-1.97-99$ & $12 \mathrm{~B}$ & Mott. gr. & Plumose-sheaf & ++ & $\begin{array}{l}+\rightarrow \mathrm{Sa}_{\mathrm{G}}, \mathrm{C}-\mathrm{N} \\
\text { Ge }\end{array}$ & + & -- & $1-5$ & $\pi$ & & & \\
\hline
\end{tabular}

Notes: Colors: Br. or br. = brown. Brsh. = brownish, Gr. or gr. = gray, Grsh. = grayish, and Mott. = mottled. Textures: Intergr. $=$ intergranular, Suboph. $=$ subophitic, and Branch. = branching. Mineral: $\mathrm{Ca}=$ calcite, $\mathrm{Cpx}=$ clinopyroxene, $\mathrm{C}-\mathrm{N}=$ celadonitic minerals, $\mathrm{Go}=$ goethite, $\mathrm{Id}=$ iddingsite, $\mathrm{Mc}=$ marcasite, $\mathrm{OI}=$ olivine, $\mathrm{Ph}=$ phillipsite, $\mathrm{PI}=$ plagioclase,

Py $=$ pyrite. $\mathrm{Sa}=$ saponite, and $\mathrm{Sp}=$ spinel. Amounts: $--=$ exceptional $;-=$ scarce, $+=$ present, and $++=$ abundant. Veins: Vein notation defined as $1: \mathrm{h}-0.03=$ one hairlike to 0.03 -mm-thick vein. Arrow $(\rightarrow)=$ altered to.

"Reddish brown halo along the vein located at one side of sample.

'The calcite-iddingsite vein crosscuts and thus is later than the saponite-iddingsite vein.

'Rare plumose texture observed locally.

${ }^{\mathrm{d}}$ Rare small vesicles filled with saponite and pyrite.

${ }^{\text {e}}$ Probably primary sulfide droplets attached unto skeletal magnetite.

Rare small vesicles filled with saponite.

${ }^{\mathrm{g}}$ Rare small vesicles filled with saponite and iddingsite.

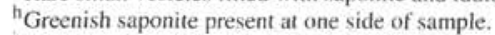

'This sample is criss-crossed by several veins.

Iddingsite mainly localized to one side of sample (halo?).

${ }^{k}$ Celadonitic mineral alone or grading into saponite.

'Branching vein.

m Brown halo at one side of sample; stained plagioclases near vein. 
Table 1 (continued).

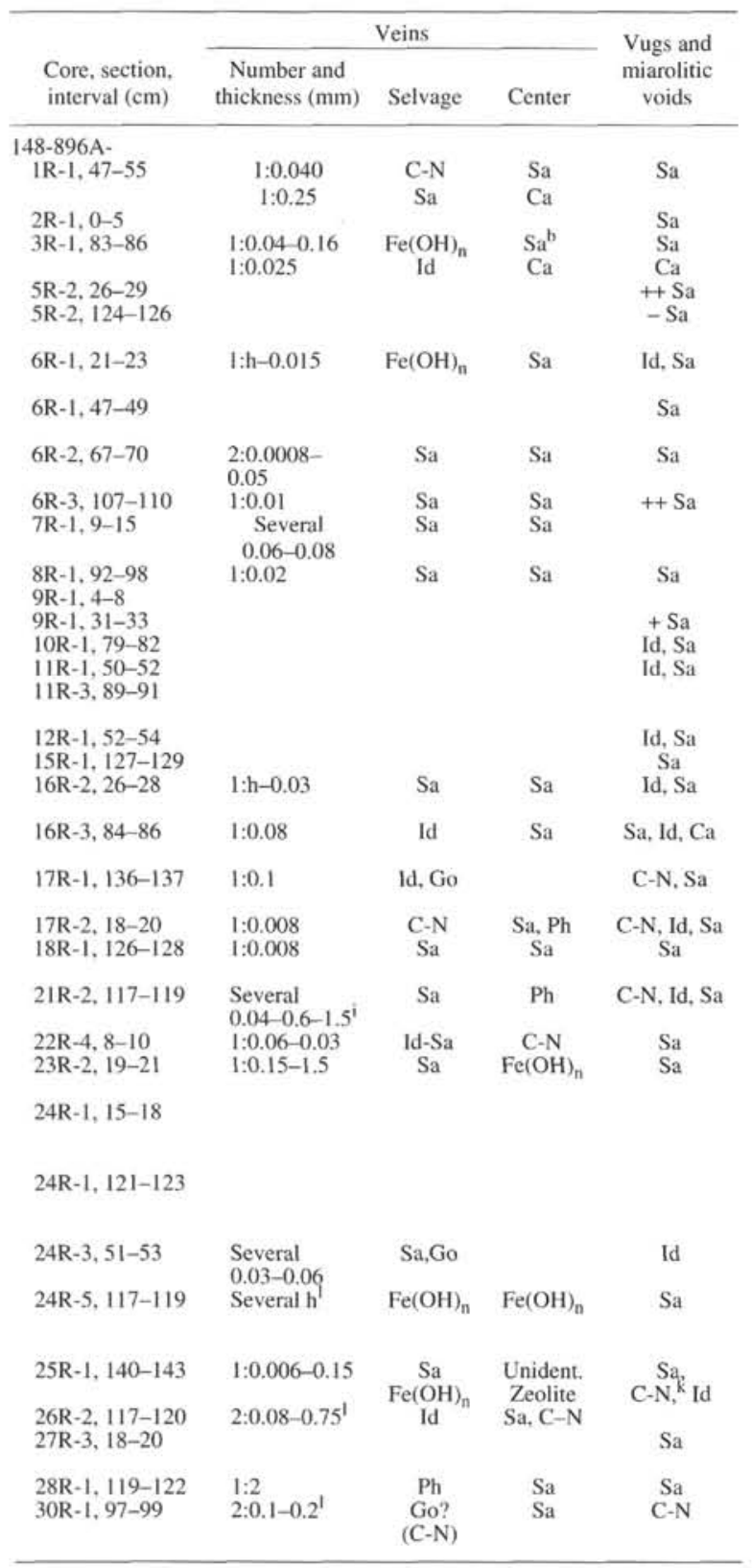

between observed secondary mineralogy and chemical composition could result from sample heterogeneity with respect to alteration mineral distribution. In the five cases mentioned, the sample portions analyzed for chemistry had to contain some carbonate because their $\mathrm{CO}_{2}$ content was high, but the mineral was probably missing from the slabs that were sawed off to prepare the thin sections.

The samples containing abundant olivine relicts (148-896A-24R$1,15-18 \mathrm{~cm}$, Piece $1 ; 24 \mathrm{R}-1,51-53 \mathrm{~cm}$, Piece 5; 24R-5, 117-119 cm, Piece 12; 5R-2, 124-126 cm, Piece 3; 6R-1, 47-49 cm, Piece 5B; and $11 \mathrm{R}-3,89-91 \mathrm{~cm}$, Piece 15) are chemically different from the average sample with respect to both their oxidation ratio and $\mathrm{K}_{2} \mathrm{O}$ content (see below).

\section{$\mathrm{K}_{2} \mathrm{O}$}

Potassium content is thought to be one of the good indicators of low-temperature alteration of oceanic basalts by seawater (see dis- cussion in Honnorez, 1981). In the study samples, $\mathrm{K}_{2} \mathrm{O}$ generally ranges from $0.02 \%$ to $0.16 \%$, and only four samples have $\mathrm{K}_{2} \mathrm{O}$ values markedly higher. The samples with the two highest $\mathrm{K}_{2} \mathrm{O}$ contents (Samples 148-896A-17R-2, 18-20 cm, Piece 37, with $0.26 \%$ and $30 \mathrm{R}-1,97-99 \mathrm{~cm}$, Piece $12 \mathrm{~B}$, with $0.24 \% \mathrm{~K}_{2} \mathrm{O}$ ) contain the largest amounts of celadonitic minerals, which is the secondary phase rich in potassium. On the other hand, we did not observe any green clay minerals in the third and fourth richest samples in $\mathrm{K}_{2} \mathrm{O}$ (148-896A-7R-1, 9-15 cm, Piece 3, with $0.23 \%$ and $16 \mathrm{R}-2,26-28 \mathrm{~cm}$, Piece $1 \mathrm{C}$, with $0.19 \% \mathrm{~K}_{2} \mathrm{O}$ ). Reciprocally, several other samples contain celadonitic minerals in veins and/or vugs even though their $\mathrm{K}_{2} \mathrm{O}$ content remains within the normal range of $0.02 \%$ to $0.16 \%$. The two samples with the lowest $\mathrm{K}_{2} \mathrm{O}$ contents (148-296A-24R-1, 15-18 cm, Piece 1, and $24 \mathrm{R}-1,121-123 \mathrm{~cm}$, Piece 8, with $0.02 \% \mathrm{~K}_{2} \mathrm{O}$ ) are the subophitic basalts with almost completely fresh olivine which is the primary mineral the most sensitive to low-temperature alteration. Electron microprobe analysis of 158 fresh glass samples from pillow rims from Hole $896 \mathrm{~A}$ (Fisk et al., this volume) indicates that the $\mathrm{K}_{2} \mathrm{O}$ content of unaltered tholeiites from Hole $896 \mathrm{~A}$ ranges from $0.01 \%$ to $0.03 \%$ with a calculated average of $0.017 \%$. Two samples out of the 158 were not considered in the above average range because they had anomalously high $\mathrm{K}_{2} \mathrm{O}$ contents $(0.06 \%$ and $0.17 \%$ for Samples 148 896A-25R-2, 61-64 cm, Piece 1, and 5R-2, 32-34 cm, Piece 1C, respectively). According to the average $\mathrm{K}_{2} \mathrm{O}$ content of glass samples with low potassium contents, such as 148-896A-24R-1, 15-18 cm (Piece 1), and 24R-1, 121-123 cm (Piece 8), which contain only $0.02 \% \mathrm{~K}_{2} \mathrm{O}$, are considered the least altered or almost completely unaltered rocks of the studies samples. Two of the 64 samples chemically analyzed by the shipboard scientific party (see table 6 in Alt, Kinoshita, Stokking, et al., 1993) have a potassium content lower than $0.01 \% \mathrm{~K}_{2} \mathrm{O}$, whereas the two samples with potassium content higher than $0.02 \% \mathrm{~K}_{2} \mathrm{O}$ were considered by the scientific party as altered at low temperature.

\section{$\mathrm{H}_{2} \mathrm{O}$}

The water content of the studies samples ranges from $0.70 \%$ to $2.65 \%$, which confirms the petrographic observations that all of the studies samples have been somewhat altered at low temperature. However, one-half the studies samples have $\mathrm{H}_{2} \mathrm{O}$ content between $1.28 \%$ and $1.62 \%$, and $83 \%$ of the samples have $\mathrm{H}_{2} \mathrm{O}$ content ranging from $0.96 \%$ to $1.91 \%$. The sample with the highest water content (Sample 148-896A-25R-1, 140-143 cm, Piece 17B), with $2.65 \%$ $\mathrm{H}_{2} \mathrm{O}$, and that with the third highest water content (Sample 148896 A-21R-2, $117-120 \mathrm{~cm}$, Piece 11B) with $2.13 \%$, are the rocks with the largest amounts of saponite pseudomorphs after plagioclase and, of course, after olivines as is typical for most of the study samples. Moreover, these same two samples are crossed by smectite veins up to $1.5 \mathrm{~mm}$ thick and contain numerous saponite-filled vugs. On the other hand, the second richest sample in $\mathrm{H}_{2} \mathrm{O}$ (148-896A-28R-1, 119-122 cm, Piece 18D, with $2.32 \% \mathrm{H}_{2} \mathrm{O}$ ) contains a brown halo along a saponite vein, but is not otherwise distinguished with respect to alteration. However, other samples containing either numerous saponite-filled vugs (148-896A-9R-1, 34-33 cm, Piece 6) or several thin or one thick vein and vugs (148-896A-7R-1, 9-15 cm, Piece 3; 23R-2, 19-21 cm, Piece 2A; and 26R-2, 117-120 cm, Piece 18B) do not have particularly high water contents $(1.82 \%, 1.55 \%$, and $1.75 \%$, respectively). Finally, the two subophitic samples (148-896A-24R-1, $15-18 \mathrm{~cm}$, Piece 1 , and $24 \mathrm{R}-1,121-122 \mathrm{~cm}$, Piece 8 ) with the smallest amount of secondary minerals (they contain almost completely fresh olivine and titanomagnetite) and the lowest $\mathrm{K}_{2} \mathrm{O}$ contents and oxidation ratios have average water contents of $1.40 \%$ and $1.44 \%$, respectively. As we will see, $\mathrm{H}_{2} \mathrm{O}$ content is not always a good indicator of alteration degree.

No systematic correlation exists between the use of $\mathrm{K}_{2} \mathrm{O}$ and $\mathrm{H}_{2} \mathrm{O}$ contents as criteria of low-temperature alteration of oceanic basalt (Fig. 1). If it were always true that increases in both these contents in- 
Table 2. Representative electron microprobe analyses of clay minerals and iddingsite from Hole 896A.

\begin{tabular}{|c|c|c|c|c|c|c|c|c|c|c|c|c|c|c|c|c|c|c|}
\hline Mineral: & Sa & Sa & Sa & Sa & $\mathrm{C}-\mathrm{N}$ & $\mathrm{C}-\mathrm{N}$ & $\mathrm{C}-\mathrm{N}$ & $\mathrm{C}-\mathrm{N}$ & $\mathrm{C}-\mathrm{N}$ & $\mathrm{C}-\mathrm{N}$ & C-N Sa & C-N Sa & C-N Sa & Id & Id & Id & Id & Id \\
\hline Mineral type: & 1 & 1 & 2 & 2 & 3 & 3 & 3 & 4 & 4 & 4 & 5 & 5 & 5 & $8 \mathrm{a}$ & $8 \mathrm{a}$ & $8 b$ & $8 b$ & $8 \mathrm{~b}$ \\
\hline Core, section: & $24 \mathrm{R}-1$ & $25 \mathrm{R}-3$ & $4 \mathrm{R}-\mathrm{I}$ & $2 \mid R-2$ & $17 \mathrm{R}-3$ & $17 \mathrm{R}-3$ & $21 \mathrm{R}-1$ & $17 \mathrm{R}-4$ & $17 \mathrm{R}-4$ & $23 \mathrm{R}-2$ & $4 \mathrm{R}-1$ & $2 \mid R-1$ & $25 \mathrm{R}-3$ & $17 R-4$ & $23 R-1$ & $17 R-4$ & $17 \mathrm{R}-4$ & $23 R-2$ \\
\hline Interval $(\mathrm{cm})$ : & $88-92$ & $30-34$ & $37-44$ & $33-38$ & 43-45 & $43-45$ & $84-91$ & $15-20$ & $15-20$ & $0-6$ & $37-44$ & $84-91$ & $30-34$ & $15-20$ & $0-6$ & $15-20$ & $15-20$ & $0-6$ \\
\hline Piece no.: & 6 & 5 & 8 & 4 & 4 & 4 & 14 & $2 \mathrm{c}$ & $2 \mathrm{c}$ & 1a & 8 & 14 & 5 & $2 \mathrm{c}$ & la & $2 c$ & $2 \mathrm{c}$ & la \\
\hline Prof (mbsf): & 402.7 & 414.4 & 219.3 & 374.8 & 346.8 & 346.8 & 373.7 & 348.1 & 348.1 & 393.6 & 219.3 & 373.7 & 414.4 & 348.1 & 393.6 & 348.1 & 348.1 & 393.6 \\
\hline Rock type: & Gr. adj & $\mathrm{CRH} \mathrm{A}$ & Gr. adj & Br. H & BI. H & BI. H & $\mathrm{CRH} \mathrm{A}$ & CRH A & CRH A & $\mathrm{CRH} \mathrm{A}$ & Br. H & $\mathrm{CRH} \mathrm{A}$ & $\mathrm{CRH} A$ & CRH B & CRH A,B & CRH B & $\mathrm{CRH} A$ & $\mathrm{CRHC}$ \\
\hline Occurrence: & Ol. c & Glass & Ol. & Pl. & Ol. $\mathbf{r}$ & Ol. $\mathrm{r}$ & Ves, $\mathrm{r}$ & Interst. & $\mathrm{Ol} \cdot \mathrm{r}$ & Ves. & Ves. & Interst. & Ves. & Ves. & Interst. & Ves. & Ol. $\mathrm{r}$ & Interst. \\
\hline \multicolumn{19}{|c|}{ Major elements (wt\%): } \\
\hline $\mathrm{SiO}_{2}$ & 40.37 & 52.36 & 47.22 & 37.82 & 52.12 & 50.30 & 43.67 & 49.72 & 50.04 & 50.84 & 43.23 & 46.21 & 46.09 & 38.76 & 36.30 & 22.42 & 12.98 & 12.51 \\
\hline $\mathrm{Al}_{2} \mathrm{O}_{3}$ & 8.03 & 3.63 & 5.28 & 13.7 & 1.76 & 3.59 & 3.46 & 1.32 & 3.81 & 3.07 & 5.79 & 6.04 & 4.23 & 3.11 & 2.87 & 2.98 & 3.60 & 1.45 \\
\hline $\mathrm{FeOt}$ & 9.93 & 2.59 & 10.67 & 11.97 & 24.23 & 25.26 & 32.40 & 26.76 & 22.71 & 24.67 & 25.64 & 22.39 & 21.11 & 36.82 & 38.34 & 51.34 & 57.23 & 61.94 \\
\hline $\mathrm{MnO}$ & 0.00 & 0.00 & 0.00 & 0.00 & 0.00 & 0.84 & 0.19 & 0.00 & 0.00 & 0.00 & 0.00 & 0.00 & 0.00 & 0.00 & 0.00 & 0.00 & 0.00 & 0.10 \\
\hline $\mathrm{MgO}$ & 25.76 & 25.62 & 19.76 & 20.52 & 6.05 & 5.22 & 3.90 & 3.80 & 5.46 & 5.72 & 6.8 & 9.92 & 11.22 & 3. & 4.05 & 6.58 & 2.77 & 5.02 \\
\hline $\mathrm{CaO}$ & 1.94 & 0.85 & 0.44 & 0.67 & 0.45 & 0.60 & 1.04 & 0.40 & 0.94 & 0.66 & 1.28 & 1.11 & 1.08 & 0.70 & 0.71 & 0.93 & 0.88 & 0.80 \\
\hline $\mathrm{Na}_{2} \mathrm{O}$ & 0.29 & 0.14 & 1.19 & 0.34 & 0.32 & 0.48 & 0.28 & 0.15 & $\begin{array}{l}0.94 \\
0.20\end{array}$ & 0.59 & 0.32 & 1.37 & 0.53 & 0.27 & 0.48 & 0.10 & 0.25 & 0.34 \\
\hline $\mathrm{K}_{2} \mathrm{O}$ & 0.11 & 0.19 & 0.35 & 0.16 & 8.06 & 6.77 & 6.11 & 8.56 & 6.05 & 6.42 & 3.75 & 2.37 & 3.63 & 5.20 & 4.38 & 0.62 & 0.17 & 0.04 \\
\hline $\mathrm{TiO}_{2}$ & 0.00 & 0.00 & 0.00 & 0.00 & 0.02 & 0.04 & 0.04 & 0.00 & 0.00 & 0.00 & 0.00 & 0.00 & 0.00 & 0.00 & 0.00 & 0.00 & 0.00 & 0.15 \\
\hline $\mathrm{Cr}_{2} \mathrm{O}_{3}$ & 0.00 & 0.00 & 0.00 & 0.00 & 0.00 & 0.0 & 0.00 & 0.00 & 0.00 & 0.00 & 0.00 & 0.00 & 0.00 & 0.00 & 0.00 & 0.00 & 0.00 & 0.06 \\
\hline Total & 86.42 & 85.39 & 84.90 & 85.17 & 93.00 & 93.10 & 91.09 & 90.70 & 89.20 & 91.98 & 86.84 & 89.41 & 87.89 & 87.97 & 87.14 & 84.96 & 77.88 & 82.40 \\
\hline \multicolumn{19}{|c|}{ Trace elements (ppm): } \\
\hline $\mathrm{Si}$ & & 3.77 & 3.61 & & & & 3.64 & 4.03 & 3.96 & 3.95 & 3.59 & 3.61 & 3.67 & & & & & \\
\hline $\mathrm{Al} 4$ & 0.72 & 031 & 3.01 & 1.25 & 0. & 0 & 0 & 0.13 & 0.3 & 0. & 0. & 0. & 0. & & & & & \\
\hline $\mathrm{Al}$ & 0.0 & 0.0 & & 0 & 0 & & 0 & 0. & 0. & 0 & 0 & 0. & 0. & & & & & \\
\hline $\mathrm{Al}$ & 0. & 0 & & 1. & & & & & & & 0. & 0. & 0.4 & & & & & \\
\hline $\mathrm{Fet}$ & 0. & & 0. & 0. & I. & I. & & & & I. & I. & 1. & 1.4 & & & & & \\
\hline $\mathrm{Mr}$ & 0. & & & & 0. & & & & & & 0. & 0. & 0. & & & & & \\
\hline $\mathrm{M}_{3}$ & 2. & & & & 0 . & & & & & & & 1. & 1. & & & & & \\
\hline $\mathrm{Ca}$ & 0. & & & & 0. & & & & 0 . & 0. & & 0. & 0.0 & & & & & \\
\hline $\mathrm{Na}$ & 0.0 & 0. & 0 . & 0.05 & 0. & 0. & 0. & 0. & 0. & 0. & 0. & 0. & 0.08 & & & & & \\
\hline $\mathrm{K}$ & 0.01 & 0.02 & 0.03 & 0.02 & 0.79 & 0.67 & 0.65 & 0.03 & 0.08 & 0. & 0. & 0. & 0.37 & & & & & \\
\hline$\widehat{T i}_{\mathrm{i}}$ & 0.00 & 0.00 & 0.00 & 0.00 & 0.00 & 0.00 & 0.00 & 0.02 & 0.03 & 0. & 0. & 0. & 0. & & & & & \\
\hline $\mathrm{Cr}$ & 0.00 & 0.00 & 0. & 0.00 & 0. & 0. & 0. & 0. & 0. & 0. & 0. & 0. & 0 & & & & & \\
\hline Total & 7.58 & 7.09 & 7.26 & 7.47 & 7.32 & 7.31 & 7.53 & 7.36 & 7.18 & 7.27 & 7.35 & 7.33 & 7.35 & & & & & \\
\hline
\end{tabular}

Notes: Data from Laverne et al. (this volume), Formulas are calculated on the basis of 22 oxygens. Gr. adj, = gray rock adjacent to a halo; $\mathrm{CRH} A, \mathrm{CRH} B$, and $\mathrm{CRH} C=\mathrm{A}, \mathrm{B}$, or $\mathrm{C}$ zones of composite red halo; $\mathrm{Br}$. or Bl. $\mathrm{H}=$ brown or black halo, respectively; Ol. c or Ol. $\mathbf{r}=$ olivine core or rim, respectively; $\mathrm{Pl} .=$ plagioclase, Ves. = vesicle, and Interst. $=$ interstitial. 
Table 3. Bulk-rock chemical compositions in weight $\%$ oxides.

\begin{tabular}{|c|c|c|c|c|c|c|c|c|c|c|c|c|c|c|c|c|}
\hline $\begin{array}{l}\text { Core, section, } \\
\text { interval }(\mathrm{cm})\end{array}$ & $\begin{array}{c}\text { Piece } \\
\text { no. }\end{array}$ & $\mathrm{SiO}_{2}$ & $\mathrm{TiO}_{2}$ & $\mathrm{Al}_{2} \mathrm{O}_{3}$ & $\mathrm{Fe}_{2} \mathrm{O}_{3}{ }^{\mathrm{T}}$ & $\mathrm{MnO}$ & $\mathrm{MgO}$ & $\mathrm{CaO}$ & $\mathrm{Na}_{2} \mathrm{O}$ & $\mathrm{K}_{2} \mathrm{O}$ & $\mathrm{P}_{2} \mathrm{O}_{5}$ & $\mathrm{H}_{2} \mathrm{O}$ & $\mathrm{CO}_{2}$ & Sum & $\mathrm{FeO}$ & $\mathrm{O}^{\circ}{ }_{x}$ \\
\hline \multicolumn{17}{|l|}{$148.896 \mathrm{~A}-$} \\
\hline $2 \mathrm{R}-1,0-5$ & 1 & 47.4 & 0.75 & 17.6 & 9.72 & 0.13 & 6.98 & 12.72 & 1.78 & 0.13 & 0.13 & 1.66 & 0.65 & 99.64 & 3.51 & 0.60 \\
\hline $3 R-1,83-86$ & $9 \mathrm{~A}$ & 48.3 & 0.71 & 16.6 & 9.78 & 0.16 & 7.62 & 13.39 & 1.46 & 0.06 & 0.05 & 1.68 & 0.17 & 100.00 & 4.62 & 0.47 \\
\hline $5 R-2,26-29$ & IC & 48.2 & 0.76 & 17.2 & 9.13 & 0.17 & 7.10 & 13.38 & 1.54 & 0.06 & 0.05 & 1.67 & 0.17 & 99.38 & 4.40 & 0.46 \\
\hline $5 R-2,124-126$ & 13 & 48.7 & 0.75 & 16.8 & 9.14 & 0.16 & 7.64 & 13.36 & 1.58 & 0.09 & 0.05 & 1.28 & 0.16 & 99.74 & 4.93 & 0.40 \\
\hline $6 \mathrm{R}-1,47-49$ & $5 B$ & 47.8 & 0.74 & 16.5 & 9.30 & 0.17 & 7.92 & 13.27 & 1.61 & 0.08 & 0.05 & 1.40 & 0.21 & 99.02 & 4.88 & 0.42 \\
\hline $6 R-2,67-70$ & 8 & 48.3 & 0.76 & 17.0 & 9.43 & 0.17 & 7.38 & 13.34 & 1.46 & 0.07 & 0.05 & 1.99 & 0.35 & 100.25 & 4.98 & 0.41 \\
\hline $6 \mathrm{R}-3,107-110$ & 21 & 48.2 & 0.74 & 16.4 & 9.61 & 0.17 & 8.41 & 13.27 & 1.38 & 0.07 & 0.05 & 1.24 & 0.24 & 99.76 & 5.61 & 0.35 \\
\hline $7 R-1,9-15$ & 3 & 48.5 & 0.79 & 17.6 & 8.49 & 0.13 & 6.80 & 12.17 & 1.87 & 0.23 & 0.05 & 1.82 & 0.19 & 98.62 & 4.49 & 0.41 \\
\hline $8 R-1.92-98$ & 19 & 48.1 & 0.79 & 17.5 & 8.76 & 0.15 & 6.58 & 13.39 & 1.63 & 0.06 & 0.05 & 1.58 & 0.22 & 98.81 & 4.02 & 0.49 \\
\hline $9 \mathrm{R}-1,3 \mathrm{I}-33$ & 6 & 47.4 & 0.75 & 16.4 & 9.59 & 0.20 & 8.33 & 12.65 & 1.42 & 0.08 & 0.05 & 1.91 & 0.20 & 98.92 & 5.10 & 0.41 \\
\hline 10R-1. 79-82 & $8 \mathrm{C}$ & 47.7 & 0.77 & 16.7 & 9.47 & 0.19 & 7.62 & 13.07 & 1.48 & 0.10 & 0.06 & 1.80 & 0.19 & 99.10 & 4.97 & 0.42 \\
\hline IIR-1.50-52 & $3 \mathrm{E}$ & 47.8 & 0.75 & 16.4 & 9.90 & 0.18 & 7.63 & 13.08 & 1.48 & 0.13 & 0.07 & 1.31 & 0.19 & 98.89 & 4.98 & 0.44 \\
\hline IIR-3, 89-91 & 15 & 48.6 & 0.75 & 16.5 & 9.32 & 0.17 & 7.52 & 13.21 & 1.45 & 0.03 & 0.05 & 1.14 & 0.16 & 98.94 & 5.60 & 0.33 \\
\hline $12 R-1.52-54$ & 8 & 47.4 & 0.77 & 16.4 & 10.11 & 0.17 & 7.10 & 13.09 & 1.67 & 0.11 & 0.07 & 1.76 & 1.17 & 99.86 & 4.32 & 0.52 \\
\hline $15 \mathrm{R}-1,127-129$ & 18 & 47.8 & 0.81 & 17.3 & 9.37 & 0.17 & 6.72 & 13.32 & 1.58 & 0.05 & 0.05 & 1.38 & 0.16 & 98.71 & 4.66 & 0.45 \\
\hline $16 R-2,26-28$ & IC & 47.9 & 0.78 & 16.5 & 9.59 & 0.14 & 8.06 & 11.88 & 1.76 & 0.19 & 0.06 & 1.62 & 0.20 & 98.65 & 3.91 & 0.55 \\
\hline $16 \mathrm{R}-3.84-86$ & 4 & 48.2 & 0.75 & 16.5 & 9.44 & 0.17 & 7.61 & 13.28 & 1.49 & 0.04 & 0.05 & 1.07 & 0.31 & 98.96 & 5.34 & 0.37 \\
\hline $17 R-1.136-137$ & $16 \mathrm{~A}$ & 48.5 & 0.90 & 16.1 & 9.59 & 0.17 & 7.48 & 12.88 & 1.65 & 0.14 & 0.07 & 1.00 & 0.32 & 98.85 & 5.20 & 0.40 \\
\hline $17 R-2,18-20$ & $3 \mathrm{~A}$ & 48.7 & 0.87 & 15.9 & 10.30 & 0.17 & 7.27 & 12.75 & 1.65 & 0.26 & 0.08 & 0.98 & 0.95 & 99.86 & 5.28 & 0.43 \\
\hline $18 \mathrm{R}-1.126-128$ & $11 \mathrm{~B}$ & 48.9 & 0.91 & 16.2 & 9.56 & 0.18 & 7.69 & 12.86 & 1.61 & 0.11 & 0.06 & 0.83 & 0.15 & 99.07 & 5.53 & 0.35 \\
\hline $21 R-2,117-119$ & IIB & 47.9 & 0.86 & 15.2 & 10.15 & 0.15 & 9.13 & 11.58 & 1.55 & 0.15 & 0.05 & 2.13 & 1.25 & 100.14 & 4.69 & 0.48 \\
\hline $22 R-4,8-10$ & IB & 48.5 & 0.91 & 16.0 & 10.27 & 0.18 & 7.41 & 13.05 & 1.58 & 0.10 & 0.06 & 0.96 & 0.19 & 99.23 & 5.42 & 0.41 \\
\hline $23 R-2,19-21$ & $2 \mathrm{~A}$ & 48.1 & 0.88 & 16.9 & 9.44 & 0.16 & 7.18 & 12.50 & 1.90 & 0.12 & 0.07 & 1.55 & 0.90 & 99.71 & 4.21 & 0.50 \\
\hline $24 R-1,15-18$ & 1 & 48.1 & 0.82 & 16.3 & 9.21 & 0.15 & 9.12 & 12.22 & 1.66 & 0.02 & 0.04 & 1.40 & 0.21 & 99.27 & 6.36 & 0.23 \\
\hline $24 \mathrm{R}-1,121-123$ & 8 & 47.9 & 0.79 & 16.2 & 9.10 & 0.15 & 9.27 & 12.18 & 1.63 & 0.02 & 0.05 & 1.44 & 0.21 & 98.95 & 6.30 & 0.23 \\
\hline $24 \mathrm{R}-3,5 \mathrm{I}-53$ & 5 & 48.2 & 0.89 & 16.6 & 9.54 & 0.18 & 7.45 & 12.64 & 1.86 & 0.06 & 0.06 & 1.34 & 0.17 & 98.92 & 4.42 & 0.48 \\
\hline $24 \mathrm{R}-5,117-119$ & 12 & 47.7 & 0.76 & 16.3 & 9.02 & 0.15 & 9.67 & 12.04 & 1.67 & 0.05 & 0.04 & 1.43 & 0.18 & 99.04 & 5.02 & 0.38 \\
\hline $25 \mathrm{R}-1,140-143$ & 17B & 46.2 & 0.89 & 17.1 & 10.59 & 0.17 & 7.35 & 11.66 & 1.95 & 0.16 & 0.11 & 2.65 & 0.39 & 99.24 & 3.34 & 0.65 \\
\hline $26 \mathrm{R}-2,117-120$ & $18 \mathrm{~B}$ & 47.5 & 0.89 & 17.1 & 9.78 & 0.15 & 7.15 & 12.24 & 1.95 & 0.15 & 0.09 & 1.75 & 0.29 & 99.00 & 3.87 & 0.56 \\
\hline $27 R-3,18-20$ & $3 \mathrm{~A}$ & 47.5 & 0.65 & 16.3 & 9.68 & 0.15 & 8.23 & 13.24 & 1.39 & 0.09 & 0.04 & 1.52 & 0.18 & 99.03 & 5.67 & 0.35 \\
\hline $28 \mathrm{R}-1,119-122$ & $18 \mathrm{D}$ & 46.9 & 0.64 & 16.0 & 9.71 & 0.17 & 8.96 & 12.80 & 1.29 & 0.08 & 0.04 & 2.32 & 0.40 & 99.31 & 5.35 & 0.38 \\
\hline $30 R-1,97-99$ & $12 \mathrm{~B}$ & 49.1 & 0.85 & 14.8 & 10.25 & 0.21 & 8.06 & 12.91 & 1.66 & 0.24 & 0.06 & 0.70 & 0.25 & 99.09 & 6.04 & 0.34 \\
\hline
\end{tabular}

Note: Fe oxidation ratio $\left(\mathrm{O}^{\circ}\right)$ is defined in text.

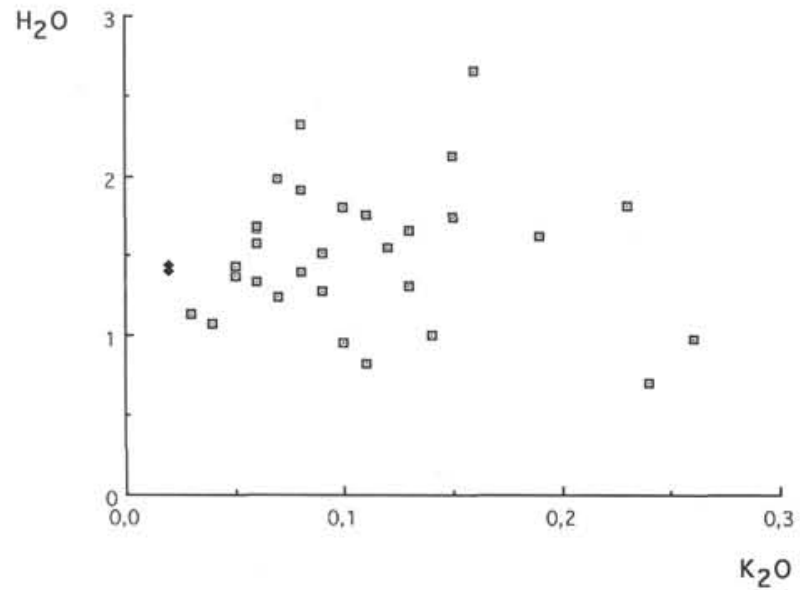

Figure 1. Bulk-rock weight oxide $\mathrm{K}_{2} \mathrm{O}$ contents vs. $\mathrm{H}_{2} \mathrm{O}$ contents (in \%) of study samples. Notice that the two highest $\mathrm{K}_{2} \mathrm{O} \%$ values do not correspond to samples with the highest $\mathrm{H}_{2} \mathrm{O}$ contents. The two samples with the lowest values of $\mathrm{O}^{\circ}$, and $\mathrm{K}_{2} \mathrm{O}$ content are the two coarse subophitic basalt Samples 148-896A-24R-1, 15-18 cm, and 24R-1, 121-123 cm, represented by diamonds (see Fig. 11).

dicate "submarine weathering," then the samples containing a high percentage of $\mathrm{K}_{2} \mathrm{O}$ should also have a high amount of $\mathrm{H}_{2} \mathrm{O}$, and vice versa. However, we do not observe such a consistent relationship between the two parameters in our study samples from Hole 896A. For instance, the study samples with the two lowest $\% \mathrm{~K}_{2} \mathrm{O}$ (148-896A$24 \mathrm{R}-1,15-18 \mathrm{~cm}$, Piece 1, and 24R-1, 121-123 cm, Piece 8), both with $0.02 \% \mathrm{~K}_{2} \mathrm{O}$, contain $1.40 \%$ and $1.44 \% \mathrm{H}_{2} \mathrm{O}$. Conversely, the study sample with the lowest $\mathrm{H}_{2} \mathrm{O}$ contents (148-896A-30R-1, 97-99 $\mathrm{cm}$, Piece 12B) contains the second highest $\mathrm{K}_{2} \mathrm{O}$ value $(0.24 \%)$. Similarly, among the samples analyzed by the scientific party (Alt, Ki- noshita, Stokking, et al., 1993), those with the lowest $\mathrm{K}_{2} \mathrm{O}$ content $(\leq 0.02 \%)$ have $\mathrm{H}_{2} \mathrm{O}$ contents ranging from $1.50 \%$ to $2.17 \%$, significantly higher than that of the least altered rocks from Hole 896A. On the other hand, samples with the lowest $\mathrm{H}_{2} \mathrm{O}$ contents (ranging from $0.37 \%$ to $0.89 \%$ ) have $\mathrm{K}_{2} \mathrm{O}$ contents ranging from $0.08 \%$ to $0.25 \%$, significantly higher than the least altered rocks from Hole 896A. The latter value is the third highest $\mathrm{K}_{2} \mathrm{O}$ content of all the rocks analyzed by the scientific party.

This discussion consistently confirms Honnorez's (1981) observation that no single chemical or mineralogical criteria can be used for measuring the degree of low-temperature alteration of seafloor basalts because of the heterogeneity of the processes involved, and that of the resulting secondary mineralogy. In oceanic basalts altered at low temperature, $\mathrm{K}$ mainly resides in celadonite, nontronite, and phillipsite. Only celadonite is sufficiently abundant in the study samples to explain an increase of their $\mathrm{K}_{2} \mathrm{O}$ content. On the other hand, $\mathrm{H}_{2} \mathrm{O}$ is present in most of the secondary minerals present in the study samples. Hence, the $\mathrm{K}_{2} \mathrm{O}$ and $\mathrm{H}_{2} \mathrm{O}$ contents depend both on secondary mineralogy, which depends on the alteration processes, but they do not necessarly correlate in all the samples. Finally, due to heterogeneity of low-temperature alteration processes in seafloor basalts, thin sections may be not as good indicators of alteration as bulk-rock chemical analyses because the former correspond to a much smaller volume of sample than the latter. Therefore chemical compositions should, in principle, be more representative of alteration changes than visual observations of secondary mineral phases.

\section{Iron}

Total iron content of the Hole 896A tholeiites increases downhole whereas $\mathrm{MgO}$ and $\mathrm{Al}_{2} \mathrm{O}_{3}$ contents appear to decrease with depth; these downhole trends are primary differentiation trends with progressively more evolved lavas downsection (Alt, Kinoshita, Stokking, et al., 1993). Total iron content of the study samples recalculated as $\mathrm{Fe}_{2} \mathrm{O}_{3}$ ranges from $8.49 \%$ to $10.59 \%$, but for $72 \%$ of 
the samples it ranges from $9 \%$ to $10 \%$. For comparison, $\mathrm{Fe}_{2} \mathrm{O}_{3} \mathrm{~T}$ ranges from $8.10 \%$ to $10.32 \%$ in 64 samples analyzed by the Shipboard Scientific Party (see table 6 in Alt, Kinoshita, Stokking, et al., 1993) and from $9.25 \%$ to $11.40 \%$ in the 158 fresh glass samples from pillow rims analyzed by electron microprobe (Fisk et al., this volume). Only two of the study samples (148-896A-7R-1, 9-15 cm, Piece 3, and 8R$1,92-98 \mathrm{~cm}$, Piece 19) have lower total iron contents $(8.49 \%$ and $8.76 \%$, respectively). Among the study samples, these two are particularly poor in $\mathrm{Fe}$ - $\mathrm{Ti}$ oxides. In the first one, exceptionally rare $\mathrm{Fe}-\mathrm{Ti}$ oxide particles, $<1 \mu \mathrm{m}$ in size, could be observed with the highest magnification $(1250 \times)$. The second sample contains a few scattered $\mathrm{Fe}$ - $\mathrm{Ti}$ oxide particles also about $1 \mu \mathrm{m}$ or less in size. It probably means that a minimum iron content threshold exists in tholeiites for titanomagnetite to form. Six other samples have higher $\mathrm{Fe}_{2} \mathrm{O}_{3}$ contents, with the highest value of $10.59 \%$ in Sample 148-896A-25R-1, $140-143 \mathrm{~cm}$ (Piece 17B). This sample also has the highest water content and the lowest silica content, and appears to contain the largest amount of smectite pseudomorphs after plagioclase. Four of the five other samples with relatively high total iron contents correspond to samples containing celadonite-nontronite, including Samples 148896A-17R-2, 18-20 cm (Piece 3A), and 30R-1, 97-99 cm (Piece $12 \mathrm{~B}$ ), which also have the highest $\mathrm{K}_{2} \mathrm{O}$ contents (see above). The iron and $\mathrm{K}_{2} \mathrm{O}$ enrichment related to the presence of celadonitic minerals has been previously noticed in "black halos" exhibited by less than 3 m.y.-old basalts (Honnorez, 1981; Laverne and Vivier, 1983; J. Honnorez and $\mathrm{C}$. Laverne, unpubl. data). The remaining sample with a higher total iron content is Sample 148-896A-12R-1, 52-54 cm (Piece 8 ), which, even though it has only $10.11 \%$, the lowest of the high $\mathrm{Fe}_{2} \mathrm{O}_{3} \mathrm{~T}$ contents, does not exhibit any petrographical nor mineralogical peculiarity.

\section{Oxidation Ratio}

The bulk-rock oxidation ratio was defined by Honnorez (1972) as:

$$
\mathrm{O}_{x}^{\circ}=\frac{\mathrm{Fe}_{2} \mathrm{O}_{3} / 80}{\mathrm{Fe}_{2} \mathrm{O}_{3} / 80+\mathrm{FeO} / 72}
$$

Assuming a $\mathrm{Fe}_{2} \mathrm{O}_{3}$ content of $1.5 \%, \mathrm{O}^{\circ}$ of fresh MORBs are smaller than 0.14 (Honnorez et al., 1978; Böhlke et al., 1981).

The oxidation ratio ranges from 0.23 in Samples 148-896A-24R$1,15-180 \mathrm{~cm}$ (Piece 1), and 24R-1, 121-123 cm (Piece 8), to 0.65 in Sample 148-896A-25R-1, 140-143 cm (Piece 17B); however, in most samples, it ranges from 0.33 to 0.50 . Samples exhibiting a gray color have low oxidation ratios (from 0.23 to 0.43 ); however, two gray mottled and two grayish brown samples also fall within this oxidation interval. The two least oxidized Section 148-896A-24R-1 samples are the two subophitic basalts, which also contain almost completely fresh olivine and have the lowest $\mathrm{K}_{2} \mathrm{O}$ content $(0.02 \%)$; however, their $\mathrm{H}_{2} \mathrm{O}$ content is within the average range. The sample with the second lowest oxidation ratio (148-896A-11R-3, 89-91 cm, Piece 15 , with $\mathrm{O}^{\circ}=0.33$ ) also has the second lowest $\mathrm{K}_{2} \mathrm{O}$ content $(0.03 \%)$. The reciprocal is not true (i.e., samples with the highest oxidation ratios are not those with the highest $\mathrm{K}_{2} \mathrm{O}$ contents, nor are the highest $\mathrm{K}_{2} \mathrm{O}$ contents found in the same samples with the highest oxidation ratios). If one excludes the three samples that have the highest $\mathrm{K}_{2} \mathrm{O}$ contents (148-896A-7R-1, 9-15 cm, Piece 3; 17R-2, 18-20 cm, Piece $3 \mathrm{~A}$; and $30 \mathrm{R}-1,97-99 \mathrm{~cm}$, Piece 12B), there is a relatively good correlation $\left(R^{2}=0.49\right)$ between $\mathrm{K}_{2} \mathrm{O}$ content and $\mathrm{O}^{\circ}{ }_{x}$, as shown in Figure 2. Finally, the sample with the highest oxidation ratio (148$896 \mathrm{~A}-25 \mathrm{R}-1,140-143 \mathrm{~cm}$, Piece $17 \mathrm{~B}$, with $\left.\mathrm{O}^{\circ}{ }_{x}=0.65\right)$ also has the highest $\mathrm{Fe}_{2} \mathrm{O}_{3}$ content $(10.59 \%)$ and the highest $\mathrm{H}_{2} \mathrm{O}$ content $(2.65 \%)$. However, the sample with the next highest oxidation ratio falls within the range of average of $\mathrm{Fe}_{2} \mathrm{O}_{3}$ and $\mathrm{H}_{2} \mathrm{O}$ contents. Both samples have the highest $\mathrm{P}_{2} \mathrm{O}_{5}$ contents.

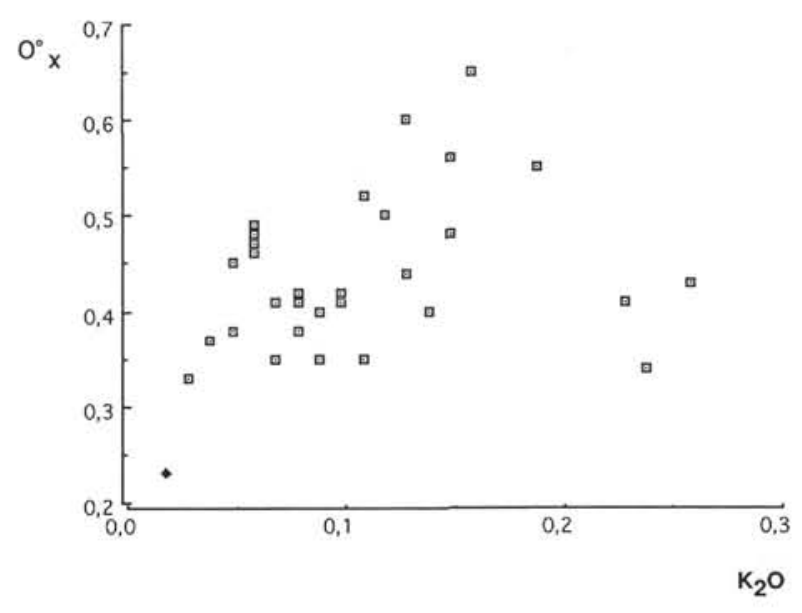

Figure 2. Bulk-rock weight oxide $\mathrm{K}_{2} \mathrm{O}$ contents (in \%) vs. Fe oxidation ratios $\left(\mathrm{O}^{\circ}\right.$, defined in text $)$ of study samples. The two samples with the lowest values of $\mathrm{O}^{\circ}$ and $\mathrm{K}_{2} \mathrm{O}$ contents are the two coarse subophitic basalt Samples 148-896A-24R-1, 15-18 cm, and $24 \mathrm{R}-1,121-123 \mathrm{~cm}$, represented by diamonds (see Fig. 11).

\section{MAGNETIC PROPERTIES}

Hysteresis parameters, Curie temperatures, and (bulk) oxidation degrees are summarized in Table 4. Ms-T curves of the studied samples are irreversible with only one exception (Sample 148-896A$24 \mathrm{R}-1,16 \mathrm{~cm}$ ). The irreversibility is an expression of the metastable nature of titanomaghemites. Unoxidized titanomagnetites have reversible thermomagnetic curves (Fig. 3). Often more than one Curie temperature can be determined for the heating curve (Fig. 4). The higher $T_{\mathrm{c}}$ is considered to belong to the decomposition product of the metastable titanomaghemite and is a Ti-poor titanomagnetite. Thus, it is not a phase that exists at ambient temperature and only the first occurring $T_{c}$ has been tabulated. The Curie temperatures of titanomagnetites and titanomaghemites increase with decreasing Ti-content and also with increasing oxidation degree. Despite the significant heterogeneity in other petrological and magnetic parameters, the $\mathrm{Cu}$ rie temperatures are remarkably constant throughout the hole, with an average value of $\mathrm{T}_{\mathrm{c}}=333 \pm 18^{\circ} \mathrm{C}(+1 \mathrm{SD})$ when the lower two values are exempted (Fig. 5). A small gradual increase of $T_{c}$ with depth can be identified at depths below $380 \mathrm{mbsf}$, although similarly high values are also present at shallow depths. When separated by lithology, pillows have slightly higher Curie temperatures than massive flows (Table 4).

The saturation magnetization values scatter more widely (Fig. 6), mainly as a result of varying magnetic mineral concentrations. Average $M_{s}$ value increases downhole: above $330 \mathrm{mbsf}, M_{\mathrm{s}}$ averages 0.37 $\pm 0.15 \mathrm{Am}^{2} / \mathrm{kg}$, whereas below $330 \mathrm{mbsf}$ it is $0.67 \pm 0.17 \mathrm{Am}^{2} / \mathrm{kg}$. Provided that the Fe-Ti oxide composition is rather constant, which it seems to be (see $T_{c}$ data), then this implies nearly twice as large a titanomaghemite concentration below 330 mbsf. The distinct increase of $M_{s}$ below $330 \mathrm{mbsf}$ is coherent with an increase in susceptibility but in contrast to a strong decrease in NRM intensities occurring below $360 \mathrm{mbsf}$ (Allerton et al., this volume). Massive basalts also average higher $M_{\text {s }}$ values than pillow lavas (Table 4) and are more abundant below $360 \mathrm{mbsf}$, but the sudden increase with depth in $M_{s}$ takes place at 330 mbsf where pillows still prevail.

As with other magnetic measurements, $M_{r s} / M_{s}$ data (Fig. 7) can be divided into two groups: one with ratios $0.2-0.45$ at depths above 330 mbsf and the other with $M_{r s} / M_{s}<0.2$ below $330 \mathrm{~m}$. The coercivity data group similarly (Fig. 8), with larger values above 330 mbsf. Rocks with stable single domain grains have a $M_{r s} / M_{s}=0.5$ and $H_{c}$ has a maximum for a given chemical composition (O'Reilly, 1984). 
Table 4. Rock magnetic properties and oxidation degree, Hole 896A.

\begin{tabular}{|c|c|c|c|c|c|c|c|}
\hline $\begin{array}{l}\text { Core, section, } \\
\text { interval }(\mathrm{cm})\end{array}$ & Lithology & $\begin{array}{l}\text { Depth } \\
\text { (mbsf) }\end{array}$ & $\begin{array}{l}T_{c} \\
\left({ }^{\circ} \mathrm{C}\right)\end{array}$ & $\begin{array}{c}M_{s} \\
\left(\mathrm{Am}^{2} / \mathrm{kg}\right)\end{array}$ & $M_{r s} / M_{s}$ & $\begin{array}{c}H_{c} \\
(\mathrm{mT})\end{array}$ & $\begin{array}{c}\text { Oxidation } \\
\text { ratio } \\
\left(\mathrm{O}^{\circ}\right)\end{array}$ \\
\hline$|\mathrm{R}-1,5|$ & P & 195.61 & 355 & 0.283 & 0.400 & 28.1 & - \\
\hline $2 \mathrm{R}-1,2$ & M & 201.10 & 315 & 0.572 & 0.220 & 8.2 & 0.60 \\
\hline $3 \mathrm{R}-1.84$ & M & 210.74 & 305 & 0.410 & 0.194 & 9.4 & 0.47 \\
\hline $5 R-2,27$ & P & 230.17 & 325 & 0.416 & 0.338 & 19.9 & - \\
\hline $5 \mathrm{R}-2,125$ & $\mathrm{P}$ & 231.15 & 315 & 0.433 & 0.343 & 18.9 & 0.40 \\
\hline $6 \mathrm{R}-1,22$ & $\mathrm{P}$ & 238.12 & 315 & 0.394 & 0.305 & 16.2 & - \\
\hline $6 \mathrm{R}-1,48$ & $\mathrm{P}$ & 238.38 & 320 & 0.411 & 0.339 & 19.5 & 0.42 \\
\hline $6 \mathrm{R}-2,68$ & P & 240.08 & 330 & 0.321 & 0.336 & 21.2 & 0.41 \\
\hline $6 \mathrm{R}-3,108$ & $\mathrm{P}$ & 241.98 & 320 & 0.230 & 0.437 & 34.0 & 0.35 \\
\hline $7 \mathrm{R}-1,12$ & $\mathrm{P}$ & 247.52 & - & 0.005 & 0.342 & 8.6 & - \\
\hline $8 \mathrm{R}-1,95$ & $\mathrm{P}$ & 258.05 & 370 & 0.290 & 0.386 & 23.7 & 0.49 \\
\hline $9 R-1,6$ & $P$ & 266.76 & - & 0.097 & 0.404 & 26.7 & - \\
\hline $9 \mathrm{R}-1.32$ & P & 267.02 & 320 & 0.471 & 0.298 & 15.7 & 0.41 \\
\hline $10 \mathrm{R}-1,80$ & M & 277.20 & 325 & 0.406 & 0.335 & 18.6 & 0.42 \\
\hline $11 R-1,51$ & P & 286.51 & 320 & 0.481 & 0.248 & 10.4 & 0.44 \\
\hline IIR-3, 90 & P & 289.90 & 320 & 0.540 & 0.263 & 9.7 & 0.33 \\
\hline $12 \mathrm{R}-1,53$ & M & 296.13 & 335 & 0.534 & 0.271 & 11.7 & 0.52 \\
\hline $15 \mathrm{R}-1,128$ & $\mathrm{P}$ & 325.58 & 360 & 0.350 & 0.371 & 22.8 & 0.45 \\
\hline $16 R-2,27$ & M & 335.67 & 320 & 1.030 & 0.096 & 4.2 & 0.55 \\
\hline $16 \mathrm{R}-3,85$ & M & 337.75 & 315 & 0.702 & 0.151 & 5.1 & 0.37 \\
\hline $17 \mathrm{R}-1,136$ & $\mathrm{P}$ & 344.86 & 330 & 0.671 & 0.193 & 7.7 & 0.40 \\
\hline $17 \mathrm{R}-2,19$ & P & 345.19 & 330 & 0.715 & 0.203 & 9.3 & 0.43 \\
\hline $18 \mathrm{R}-1,127$ & $\mathrm{P}$ & 354.37 & 325 & 0.708 & 0.187 & 7.6 & 0.43 \\
\hline $21 \mathrm{R}-2,118$ & M & 375.68 & 320 & 0.755 & 0.177 & 7.1 & 0.48 \\
\hline $22 \mathrm{R}-4,9$ & M & 388.00 & 330 & 0.895 & 0.179 & 6.6 & 0.41 \\
\hline $23 \mathrm{R}-2,20$ & M & 393.80 & 335 & 0.610 & 0.169 & 5.9 & 0.50 \\
\hline $24 \mathrm{R}-1.16$ & M & 401.96 & 209 & 0.590 & 0.073 & 2.7 & 0.23 \\
\hline $24 \mathrm{R}=1,122$ & M & 403.02 & 250 & 0.570 & 0.086 & 3.2 & 0.23 \\
\hline $24 \mathrm{R}-3,52$ & M & 405.32 & 337 & 0.847 & 0.106 & 4.2 & 0.48 \\
\hline $24 \mathrm{R}-5,118$ & M & 407.98 & 340 & 0.683 & 0.118 & 4.7 & 0.38 \\
\hline $25 \mathrm{R}-1,141$ & $\mathrm{P}$ & 412.71 & 352 & 0.308 & 0.225 & 10.7 & 0.65 \\
\hline $26 \mathrm{R}-2,118$ & P & 423.68 & 335 & 0.646 & 0.148 & 5.3 & 0.56 \\
\hline $27 \mathrm{R}-3,19$ & P & 433.69 & 355 & 0.535 & 0.197 & 11.1 & 0.35 \\
\hline $28 \mathrm{R}-1,120$ & $\mathrm{P}$ & 441.20 & 370 & 0.447 & 0.24 & 13.0 & 0.38 \\
\hline $30 R-1,98$ & $\mathrm{P}$ & 460.28 & 365 & 0.675 & 0.147 & 7.1 & 0.34 \\
\hline Average (all): & & & 326 & 0.52 & 0.244 & 12.5 & 0.43 \\
\hline$(N=34)$ & & & \pm 31 & \pm 0.22 & \pm 0.102 & 8.0 & \pm 0.09 \\
\hline Average (pillow): & & & 337 & 0.43 & 0.289 & 15.8 & 0.43 \\
\hline$(N=21)$ & & & \pm 19 & \pm 0.19 & \pm 0.088 & \pm 7.9 & \pm 0.08 \\
\hline Average (massive): & & & 310 & 0.66 & 0.167 & 7.0 & 0.43 \\
\hline$(N=13)$ & & & \pm 38 & \pm 0.18 & \pm 0.076 & \pm 4.3 & \pm 0.11 \\
\hline
\end{tabular}

Notes: Rock magnetic properties and Fe oxidation ratio $\left(\mathrm{O}^{\circ}\right)$ defined in text. $N=$ number of samples.

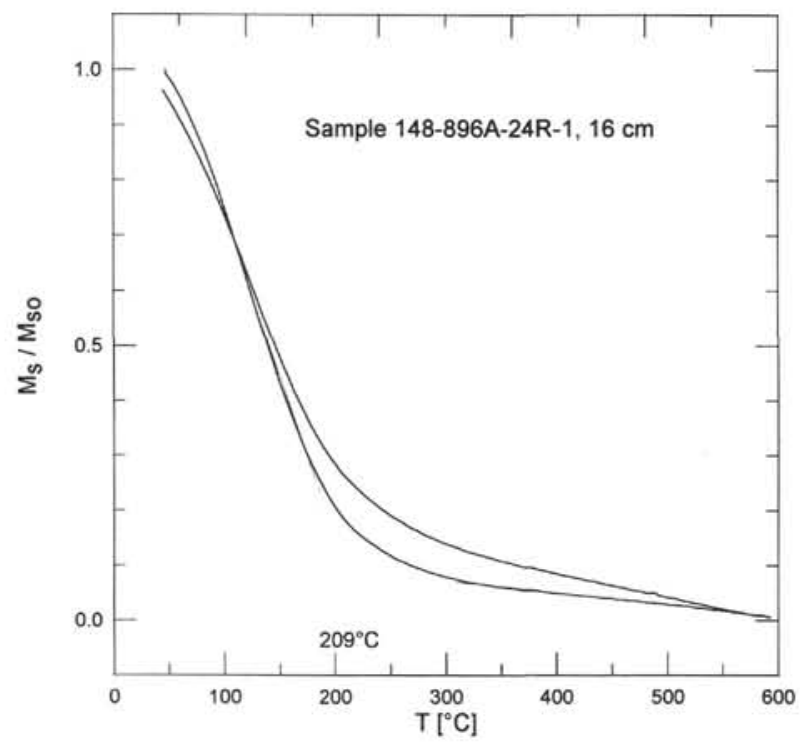

Figure 3. Thermomagnetic curve of Sample 148-896A-24R-1, $16 \mathrm{~cm}$, measuring saturation magnetization $M_{\mathrm{s}}$ vs. temperature $T$ with a heating rate of $20 \% \mathrm{~min}$ in vacuum and an applied field of $0.55 \mathrm{~T}$. The scale is normalized to initial saturation magnetization $M_{s o}$. The Curie temperature is determined to be $209^{\circ} \mathrm{C}$.

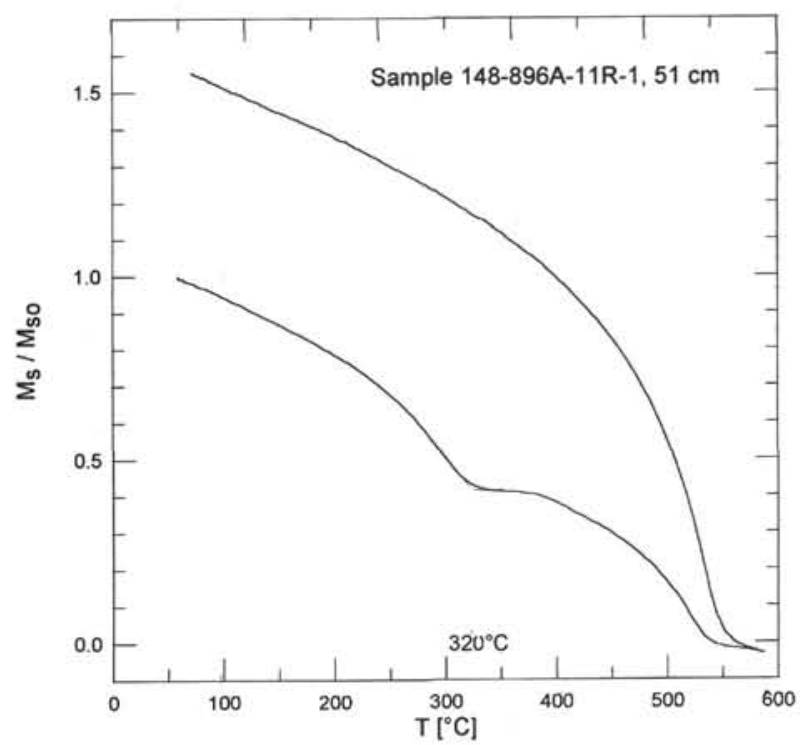

Figure 4. Thermomagnetic curve of Sample 148-896A-1IR-1, $51 \mathrm{~cm}$, measuring saturation magnetization $M$, vs. temperature $T$ with a heating rate of $20 \%$ min in vacuum and an applied field of $0.55 \mathrm{~T}$. The upper branch is the cooling curve. The scale is normalized to initial saturation magnetization $M_{x,}$. The primary Curie temperature is determined to be $320^{\circ} \mathrm{C}$.

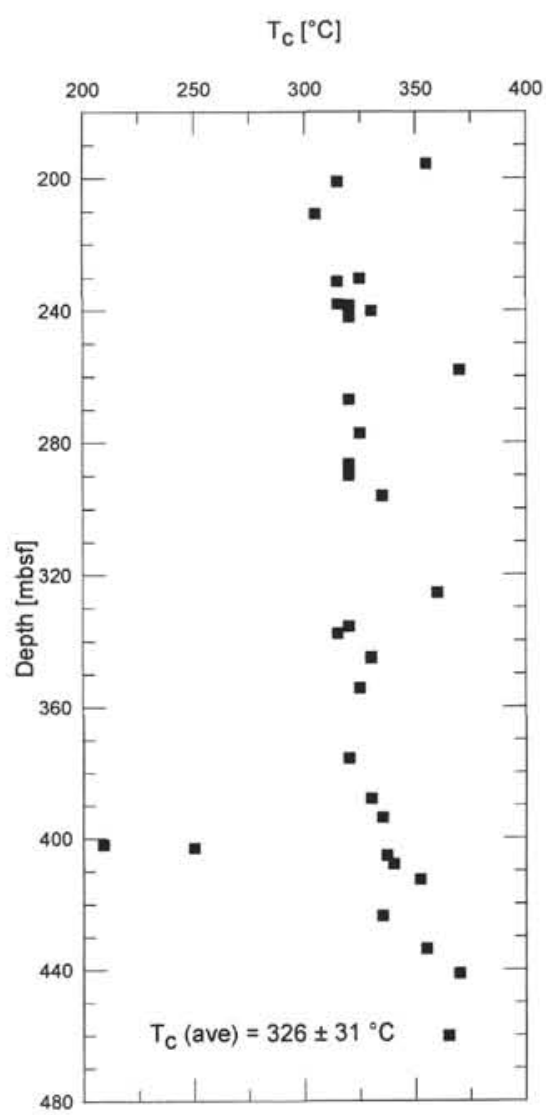

Figure 5. Curie temperatures $T_{f}$ vs. depth for Hole 896A. $T_{c}$ averages $326 \pm$ $31^{\circ} \mathrm{C}(+1 \mathrm{SD})$. When the lower two $T_{c}$ values are exempted, the average is $T_{c}$ $=333 \pm 18^{\circ} \mathrm{C}$. 


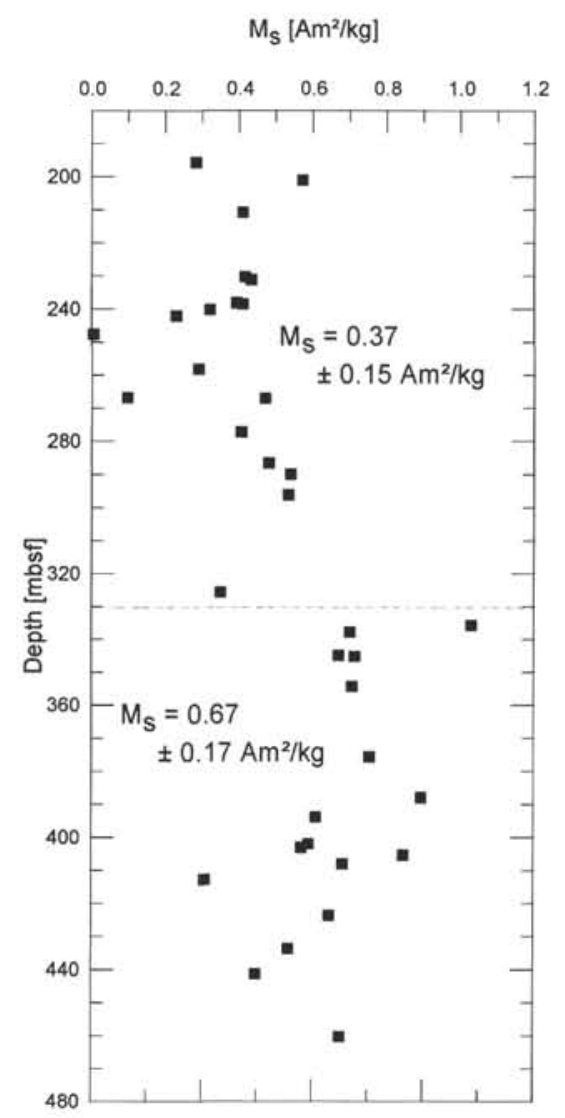

Figure 6. Saturation magnetization $M$, vs. depth for Hole 896A. Averages are given for samples from above and below $330 \mathrm{mbsf}$. The overall average is $M$. $=0.52 \pm 0.22 \mathrm{Am}^{2} / \mathrm{kg}$.

$M_{r x} / M_{x}$ ratios $>0.2$ are still considered to represent very stable magnetic properties (pseudo-single domain grains), but the magnetic hardness decreases with decreasing $M_{r s} / M_{s}$ and $H_{c}$. As for the $M_{s}$ data, the $M_{r s} / M_{c}$ ratios and $H_{c}$ values are distinctly different for pillows and massive basalts; however, the difference in rocks above and below 330 mbsf is greater than that between pillow and massive lavas.

Ac susceptibilities have been measured on ship shortly after core recovery and shore-based later in Grubenhagen and Oxford although not on identical specimens (Fig. 9). For most of the core, the average susceptibility appears to be independent of the time of measurement. However, the highest susceptibilities were measured on ship for the depth interval 360-410 mbsf whereas the average land-based measurements resulted in values about $50 \%$ lower for the same depth interval.

\section{DISCUSSION}

The relationship among rock granularity and crystallinity, and grain size of Fe-Ti oxide minerals in basaltic rocks is a well-known observational fact.

The glassy rims of pillow lavas and the hyaloclastites are holohyaline and contain a few rare intratelluric microphenocrysts of primary silicate minerals but no $\mathrm{Fe}-\mathrm{Ti}$ oxides. Such rocks are paramagnetic (e.g., Samples 148-896A-5R-1, 49-58 cm, Piece 9, and 11R-2, 30$40 \mathrm{~cm}$, Piece 3). Lavas from pillow interiors have increasing crystallinity and granularity toward the pillow center, but their $\mathrm{Fe}$-Ti oxide minerals are generally fine-grained skeletal crystals. Most of the 35 study samples fall in this category, and their Fe-Ti oxide minerals range from 0.5 to $20 \mu \mathrm{m}$ in diameter. Finally, coarse intergranular or

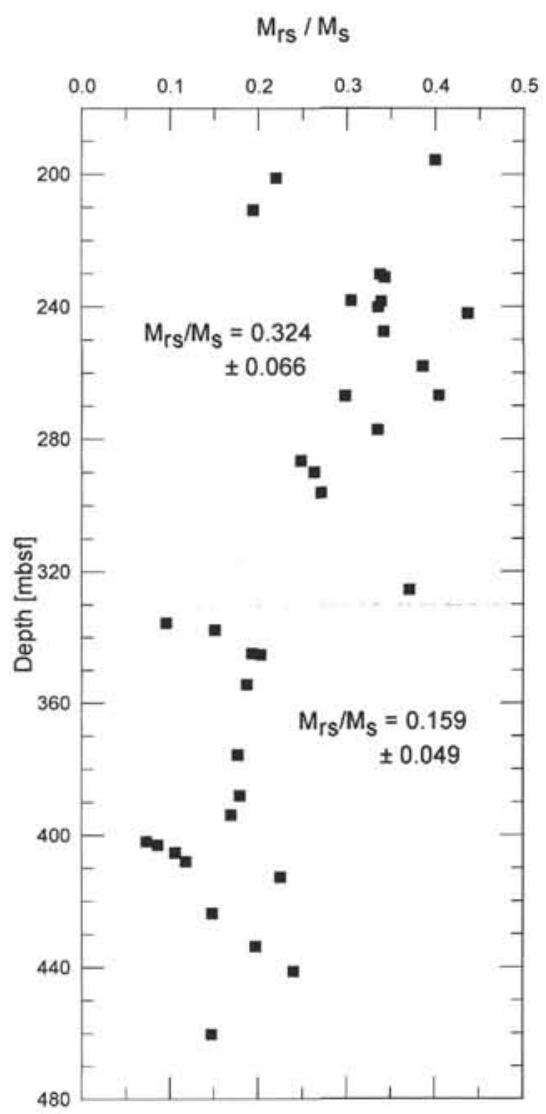

Figure 7. The ratio of saturation remanence over saturation magnetization vs. depth for Hole 896A. Averages are given for samples from above and below $330 \mathrm{mbsf}$. The overall average is $M_{i} / M_{,}=0.244 \pm 0.102$.

subophitic basalts from massive units are holocrystaline; and, in the case of the 7 study samples, their euhedral to subhedral Ti-magnetite crystals are up to $300 \mu \mathrm{m}$ in length (148-896A-1IR-3, 89-9l cm, Piece 15; 16R-2, 26-28 cm, Piece 1C; 16R-3, 84-86 cm, Piece 4; $24 \mathrm{R}-1,15-18 \mathrm{~cm}$, Piece 1; 24R-1, 121-123 cm, Piece 8; 24R-3, 51$53 \mathrm{~cm}$, Piece 5; and 24R-5, 117-119 cm, Piece 12).

The remanent magnetization of oceanic basalts resides in titanomagnetites $\left[\mathrm{Fe}_{3-r} \mathrm{Ti}_{x} \mathrm{O}_{4}(0<x<1)\right]$, which form a solid solution between magnetite and ulvöspinel. Titanomagnetites of mid-ocean ridge basalts (MORBs) have typical ulvöspinel contents of about $60 \%$ (e.g., Johnson, 1979), but significant scatter can exist in the Fe/Ti ratio of MORBs even from a single site (Bleil and Petersen, 1977). For the upper extrusive sections of Hole 504B, an average $x$-value of 0.61 has been determined (O'Donovan and O'Reilly, 1983). The Curie temperature and the saturation magnetization of titanomagnetites decrease with increasing Ti content (Stacey and Banerjee, 1974).

Titanomagnetites undergo low-temperature oxidation soon after the emplacement of the lavas on the seafloor so that unaltered titanomagnetite grains are rarely encountered in oceanic crust older than $10^{5} \mathrm{yr}$. Low-temperature oxidation in the oceanic environment proceeds by Fe migration out of the spinel lattice (Ryall and Hall, 1980; Akimoto et al., 1984; Worm and Banerjee, 1984) to result in cationdeficient titanomaghemites. "Zero age" MORBs displaying black halos that are the earliest evidence of a low-temperature alteration process (from a few degrees to about $50^{\circ} \mathrm{C}$ ) for seafloor basalts that already contain partly maghemitized titanomagnetite (Honnorez, 1981; J. Honnorez and C. Laverne, unpubl. data).

The Curie temperature increases with oxidation degree whereas saturation magnetization and lattice constant decrease (Readman and O'Reilly, 1972; Nishitani and Kono, 1983). The latter is responsible 


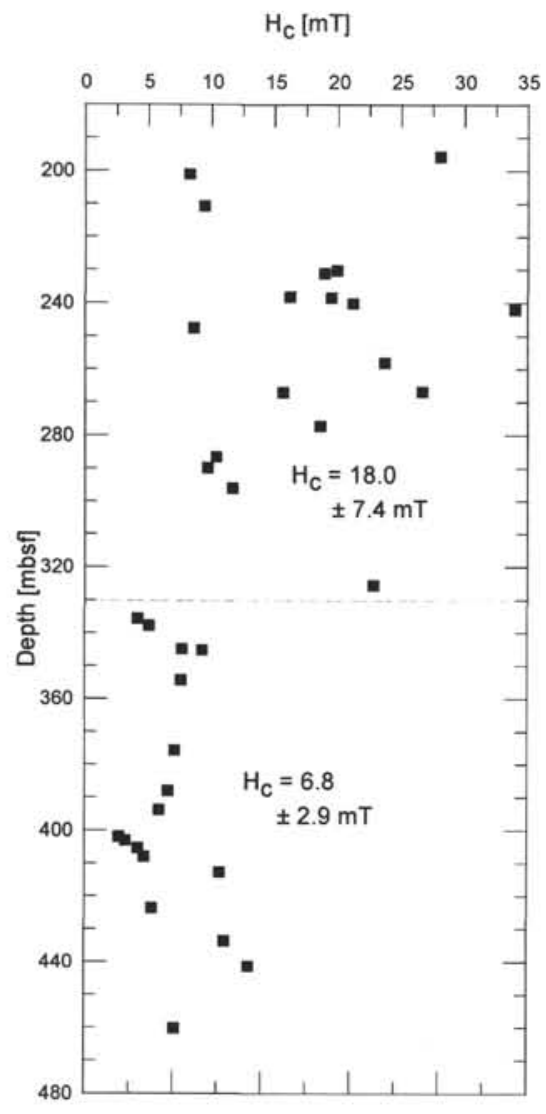

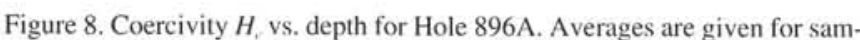
ples from above and below $330 \mathrm{mbsf}$. The overall average is $H_{c}=12.5 \pm 8.0$ $\mathrm{mT}$.

for increasing internal stress with increasing maghemitization that commonly results in shrinkage cracks of highly oxidized grains or surface areas.

Low-temperature oxidation greatly affects the intensity and magnetic stability of the NRM that is responsible for the marine lineated magnetic anomalies. Magnetic "hardness," however, also depends strongly on grain size of the ferrimagnetic phases so that a magnetic characterization should include reflected light microscopic studies of the opaque minerals present in the MORBs.

In the study samples, Curie temperatures are relatively uniform throughout Hole 896A, except for the two least-altered subophitic basalts, which have lower $T_{i}$. The average value $\left(T_{\text {ctare }}=333^{\circ} \mathrm{C}\right) \mathrm{com}$ pares well with an average of $343^{\circ} \mathrm{C}$, which was determined for the upper extrusive section of Hole 504B (Pariso and Johnson, 1991). Assuming a constant $\mathrm{Fe} / \mathrm{Ti}$ ratio for the $\mathrm{Fe}-\mathrm{Ti}$ oxides, this implies a homogeneous low-temperature oxidation process regardless of depth, lithology, and grain size. Bulk oxidation ratios of the basalts, on the other hand, show more downhole variability (Fig. 10). These differences in oxidation degree suggest different oxidation mechanisms for silicates on the one hand and for titanomagnetites on the other. Indeed, a laboratory simulation of low-temperature oxidation of titanomagnetite (Worm and Banerjee, 1984) clearly indicated that the controlling factor for aqueous oxidation is the $\mathrm{pH}$ of the solution whereas the oxygen fugacity has little effect. Acidic conditions that can occur in submarine hydrothermal systems (Seyfried and Mottl, 1982) could therefore promote maghemitization. Hence, a rather pervasive and uniform advection of low-pH, low-temperature, seawaterderived hydrothermal solutions must have altered the oceanic crust at

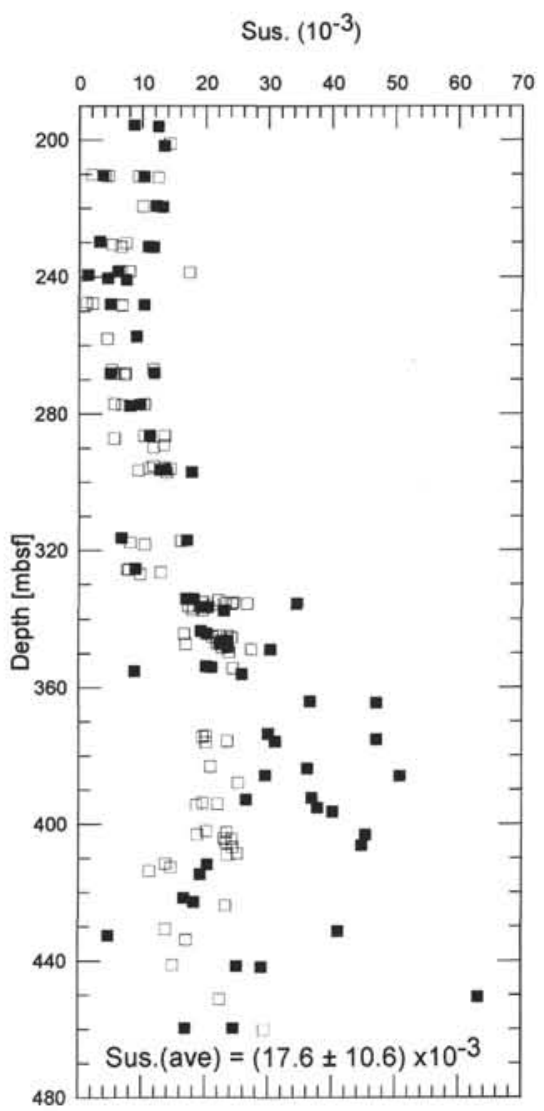

Figure 9. Magnetic susceptibility vs. depth for Hole 896A. Closed symbols indicate shipboard measurements, open symbols shore-based measurements (Oxford and Grubenhagen).

Hole 896A. Additional evidence for different oxidation mechanisms of bulk-rock and $\mathrm{Fe}-\mathrm{Ti}$ oxides can be seen in the coexistence of sulfides and (oxidized) titanomaghemites in Samples 138-896A-1 IR-3, $89-91 \mathrm{~cm}$ (Piece 15), and 16R-3, 84-86 cm (Piece 4). The timing of the maghemitization can be confined by considering the facts that titanomagnetites acquire secondary components during the oxidation process (Kelso et al., 1991) and that no other than reverse component magnetizations (except for steep drilling-induced magnetization) have been found for Hole 896A (Allerton et al., this volume; Worm et al., this volume).

Thus, one can conclude that most of the maghemitization took place before the next polarity reversal following formation of the basalts. Based on its position in the magnetic anomaly pattern (Hobart et al., 1985), where it is situated $75 \%$ across Chron $3 \mathrm{Ar}$ (with an age interval of 6.56-6.92 m.y.; Baksi, 1995), the crustal age of Hole $896 \mathrm{~A}$ can be dated to $6.83 \mathrm{~m}$.y. Thus, the maghemitization occurred within 0.27 m.y. after basalt emplacement. Other investigations on very young ocean-ridge basalts (Prévot et al., 1981; Kent and Gee, 1994) indicate an even shorter time constant by an order of magnitude for the maghemitization process.

Samples 148-896A-5R-1, 49-58 cm (Piece 9), and 11R-2, 30-40 $\mathrm{cm}$ (Piece 3), are paramagnetic because they do not contain any ferrimagnetic minerals: they are a palagonitized hyaloclastite and a fragment of glassy pillow rim with rare scattered varioles and only a few plagioclases and olivines, respectively. Similarly, Sample 148-896A7R-1, 9-15 cm (Piece 3), shows coalesced varioles and plumose textures typical of a pillow-rim variolitic zone, which also has, by far, the lowest saturation magnetization $\left(M_{s}=0.005 \mathrm{Am}^{2} / \mathrm{kg}\right)$. No opaque 
minerals were observed in these three samples. Thus, one can see that magnetic properties of these three samples are directly controlled by the lack of crystallinity in these extreme cases of quenched lavas.

On the other hand, both subophitic basalt samples of Section 148$896 \mathrm{~A}-24 \mathrm{R}-1$ have exceptionally low Curie temperatures $\left(209^{\circ}-\right.$ $250^{\circ} \mathrm{C}$ ). For instance. Sample 148-896A-24R-1, 15-18 cm (Piece 1), exhibits a reversible $M_{3}-T$ curve (see Fig. 3), a clear indication for unoxidized titanomagnetite. The low Curie temperatures correlate with the lowest porosity value $(0.6 \%)$ of all Hole $896 \mathrm{~A}$ samples (see Alt, Kinoshita, Stokking, et al., 1993, p. 171). Thus, one can conclude that the low porosity of these samples prohibited seawater-dominated conditions during hydrothermal alteration and the $\mathrm{pH}$ value did not drop so that no Fe leaching from the titanomagnetite grains (= maghemitization) occurred. As a matter of fact, both samples contain relatively large (10-200 $\mu \mathrm{m}$ in diameter), nonskeletal titanomagnetite crystals and unaltered primary sulfides.

The mineralogical composition (more particularly, the unaltered olivine and titanomagnetite) and the low $\mathrm{K}_{2} \mathrm{O}$ contents, oxidation ratios, and Curie temperatures of these samples (Fig. 11) correlate with the lowest porosity value of $0.6 \%$ measured among all the samples from Hole 896A (Alt, Kinoshita, Stokking, et al., 1993). Both samples with the lowest $T_{c}$, values are from the same section of massive basalt (148-896A-24R-1, 15-18 cm, Piece 1, and 24R-1, 121-123 $\mathrm{cm}$, Piece 8) "without red or yellow alteration halos except in Pieces 2, 3, 4, and 6" (Alt, Kinoshita, Stokking, et al., 1993). Both samples are gray rock minicores without any halos or veins, and they appear to be the freshest among the study samples: they contain the most abundant olivine relicts, the lowest oxidation ratios $(0.23)$, and the lowest $\mathrm{K}_{2} \mathrm{O}$ contents $(0,02 \%)$. At the same time, they are the coarsest rocks among the 35 study samples and display the only subophitic textures observed during this work.

On the other hand, two other samples of massive basalts from other sections of the same core (Samples 148-896A-24R-3, 51-53 cm, Piece 5 , and $24 \mathrm{R}-5,117-119 \mathrm{~cm}$, Piece 12) have high $T_{c}$ values $\left(337^{\circ}\right.$ and $340^{\circ} \mathrm{C}$, respectively). They are from parts of the same core where "nearly the whole section exhibits abundant oxidative alteration" or "most pieces are highly altered to oxidized red and black ... zones" (Alt, Kinoshita, Stokking, et al., 1993). The latter two samples exhibit brown colors and range from coarse to medium and are intergranular (however, they are finer grained than the two samples with the low $T_{c}$ values). Three other study samples (148-896A-1 1R-3, 89-91 $\mathrm{cm}$, Piece 15; 16R-2, 26-28 cm, Piece 1C; and 16R-3, 84-86 cm, Piece 4) are also massive basalts that display a coarse, intergranular texture. Their color ranges from gray to grayish brown, but the cores where they come from exhibit red and yellow alteration halos (Alt. Kinoshita, Stokking, et al., 1993). The first of these three samples contains just a few olivine relicts and no secondary mineral veins. whereas the other two do not contain any olivine but have saponite veins. The three samples have high $T_{c}$, values that range from $315^{\circ}$ to $320^{\circ} \mathrm{C}$.

The difference between the two study samples of gray massive basalts with the low values and the other study massive basalts with the high $T_{c}$ values lies in the coarser grain size, subophitic texture, and freshness of olivine in the former. All of the other massive basalts with high $T_{i}$ values studied, whether they display gray or brown colors, come from pieces or sections exhibiting red or yellow alteration halos or not, and contain secondary mineral veins or not.

Whether there is a relationship between the massive basalt coarsness of grain size and subophitic texture, on the one hand, and their freshness as expressed by the low $T$, values and the abundance in olivine relicts, on the other hand, remains to be confirmed. Two samples do not allow a generalization. However, one can see quite clearly that these two samples of massive basalts are essentially unaltered. Moreover, massive basaltic units are much less fractured and, hence, permeable than pillow lava (and, of course, breccia) units. It seems likely that the low porosity of the massive basalts from Section 148-896A$24 \mathrm{R}-\mathrm{I}$ prohibited seawater-dominated conditions during hydrother-

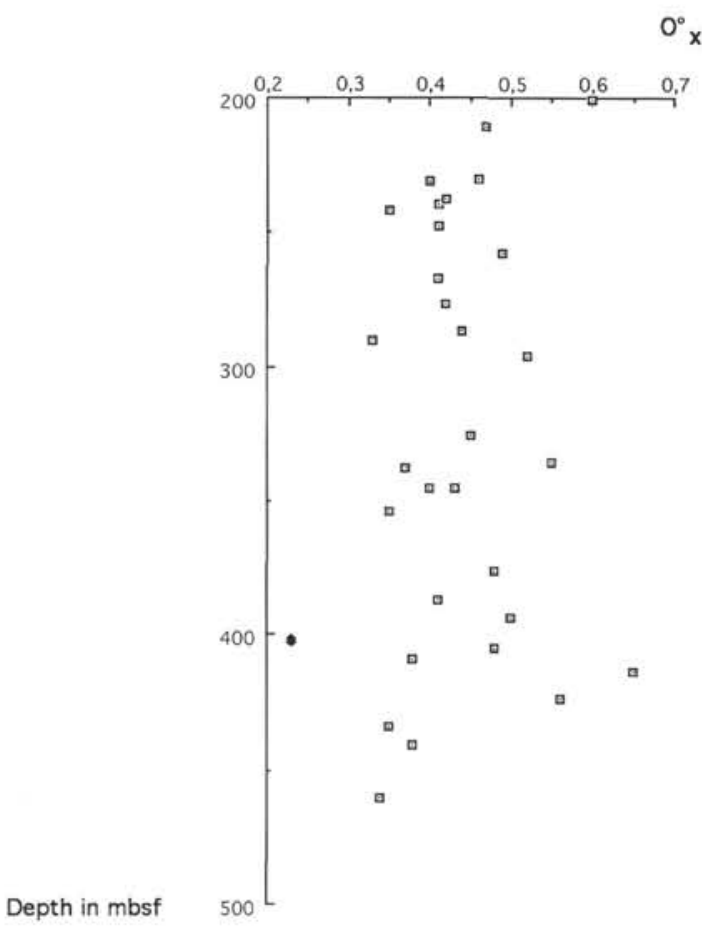

Figure 10. Bulk-rock Fe oxidation ratios $\left(\mathrm{O}_{x}^{\circ}\right.$, defined in text) vs. downhole depth (in mbsf). The two samples with the lowest $\mathrm{O}^{\circ}$, values, just below the 400 mbsf depth, are the two coarse subophitic basalt samples (148-896A$24 \mathrm{R}-1,15-18$ and $121-123 \mathrm{~cm})$, represented by diamonds.

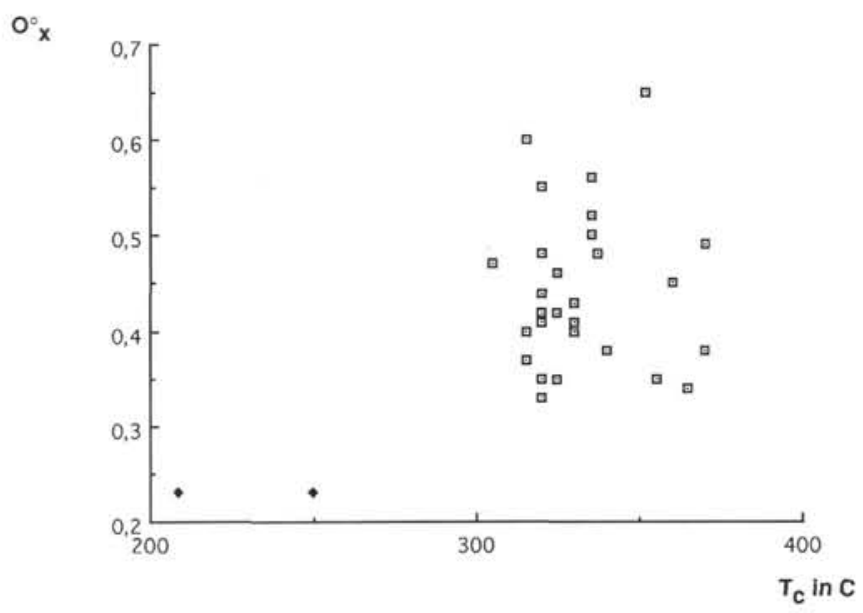

Figure 11. Bulk-rock Fe oxidation ratios $\left(\mathrm{O}^{\circ}{ }^{\circ}\right.$, defined in text) vs. Curie temperature values $\left(T_{c}\right.$ in $\left.{ }^{\circ} \mathrm{C}\right)$. The two samples with the lowest values of $\mathrm{O}^{\circ}$ and $T_{t}$ are the two coarse subophitic basalt samples (148-896A-24R-1, 15-18 cm, and $24 \mathrm{R}-1,12 \mathrm{l}-123 \mathrm{~cm}$ ), represented by diamonds (see Fig. 2).

mal alteration and, as a consequence, the $\mathrm{pH}$ did not drop so that no Fe leaching from the titanomagnetite grains (maghemitization) occurred.

The "softer" magnetic properties at depth correlate with larger grain sizes of titanomaghemite, particularly in thick flows, and not with a change in oxidation degree. However, two paradoxical observations remain to be explained:

1. The homogeneity of most of the Fe-Ti oxides observed in reflected light, except maybe in the samples from Section 148- 
$896 \mathrm{~A}-24 \mathrm{R}-1$, and the absence of shrinkage cracks in the opaque minerals is puzzling if they are titanomaghemites (as the magnetic measurements indicate).

2. The coexistence of numerous, tiny, primary sulfides in Sample 148-896A-11R-3, 89-91 cm (Piece 15), appears to contradict the fact that the observed $\mathrm{Fe}$ - $\mathrm{Ti}$ oxides are titanomaghemites resulting from the oxidation of the primary titanomagnetites. The apparent contradiction is resolved when one considers that the main factor controlling the low-temperature oxidation of the primary $\mathrm{Fe}-\mathrm{Ti}$ oxides is the $\mathrm{pH}$ of the altering solution and not the system oxygen fugacity (Worm and Banerjee, 1984).

A very striking and not previously reported phenomenon is the strong decrease with time in measured susceptibility $k$ for samples from the depth interval $360-410 \mathrm{mbsf}$. Although the reported values were not obtained on identical specimens, the much higher average of 14 shipboard results compared with the average of 18 shore-based measurements, together with similar averages in the upper depth interval, is strong evidence for a real-time dependence of susceptibility in these basalts with coarse-grained Fe-Ti oxides. A time dependence of susceptibility, termed susceptibility relaxation or magnetic aftereffect, was observed for many ferro- and ferrimagnetics when $k$ is measured following a rapid change in magnetization through alternatingfield (AF) demagnetization (e.g., Kronmüller and Walz, 1980). This aftereffect is strongly temperature dependent and results from the diffusion of interstitial atoms, vacancies, or electrons within the lattice of a magnetic crystal. Some crystal point defects tend to migrate into magnetic domain walls, thereupon impeding domain wall movement, the macroscopic observation of which is a decrease in $k$. Susceptibility relaxation has been determined for synthetic titanomagnetite and titanomaghemite (Schmidbauer and Fassbinder, 1987) and two submarine basalts with large Fe-Ti oxides from DSDP Leg 64 (Prévot and Bina, 1993). Both used AF demagnetization to initiate diffusion, which resulted in susceptibility changes of $<5 \%$. The initiating process for an aftereffect in Hole 896A basalts may have been the stress released by drilling of the core. However, the time constant (months compared to minutes in normal laboratory experiments) and the order of magnitude difference in amplitude are unprecedented for these observations on coarse-grained titanomaghemites. The magnetic aftereffect is likely to affect the stability of magnetic remanences and, therefore, the capability of oceanic rocks to preserve paleofield directions. This phenomenon will be studied further with conventional relaxation experiments on basalts from this depth interval and also on other basalts with large Fe-Ti oxides.

\section{CONCLUSIONS}

Magnetic property measurements show that all but two of the study samples, excluding the three completely or almost completely holohyaline samples which are paramagnetic, contained titanomaghemite as a ferrimagnetic phase. The only exceptions are the two low porosity, coarse subophitic basalts that contain relatively large (from 10 to $200 \mu \mathrm{m}$ ) unoxidized titanomagnetite crystals. The presence of titanomaghemite as the only ferrimagnetic mineral in most of the study samples explains the rather high, remarkably constant Curie temperatures through the entire hole, with an average value of $333^{\circ} \mathrm{C}$. This value is very similar to the $343^{\circ} \mathrm{C}$ inferred for Hole 504B upper basalts (Pariso and Johnson, 1991). This observation implies a homogeneous low-temperature alteration process for titanomagnetite of the upper few hundreds meters of the oceanic crust at both Sites 896 and 504 regardless of differences in lithology, alteration intensity and present day crustal hydrodynamics.

Saturation magnetization values scatter more than the Curie temperatures because the former are related to the amount of ferrimagnetic phases present in the sample. A downhole increase in $M_{s}$ was observed below $330 \mathrm{mbsf}$, where average $M_{\mathrm{s}}$ changes from a value of $0.37 \mathrm{Am}^{2} / \mathrm{kg}$ above $330 \mathrm{mbsf}$ to a value of $0.67 \mathrm{Am}^{2} / \mathrm{kg}$ below. Such an increase in the $M_{x}$ value suggests that there is twice as much ferrimagnetic minerals below than above $330 \mathrm{mbsf}$, assuming a rather constant chemical composition of the Fe-Ti oxides confirmed by the $T_{r}$ data. This is coherent with an increase in magnetic susceptibility and in contrast with strong NRM and coercivity decreases below 360 mbsf.

The low $\mathrm{K}_{2} \mathrm{O}$ contents, oxidation ratios, and Curie temperatures consistently appear to be good indicators of the absence of low-temperature oxidizing alteration of upper oceanic crust basalts by seawater. Samples with these characteristics, such as the two subophitic basalts containing titanomagnetite as the ferrimagnetic phase, can be petrographically distinguished from the other, more altered samples through the abundance of fresh olivine and titanomagnetite. However, the use of petrographical and/or geochemical criteria to discriminate fresh from altered rocks is limited to those few extreme cases mentioned above. For instance, the two samples with the highest $\mathrm{K}_{2} \mathrm{O}$ contents appear indeed to be petrographically those where the basaltic primary minerals have been the most intensively replaced; they also contain the largest amounts of celadonitic minerals. However they are not the most hydrated. On the other hand the samples with the next two highest $\mathrm{K}_{2} \mathrm{O}$ contents do not contain any celadonitic minerals, and conversely, several samples containing celadonitic minerals have the typical average $\mathrm{K}_{2} \mathrm{O}$ contents. Finally the $\mathrm{H}_{2} \mathrm{O}$ content of bulk-rock samples is very poorly correlated to other alteration criteria.

If one excludes the cases of the paramagnetic samples that are the poorest in $\mathrm{Fe}$ - Ti oxides and have the lowest $\mathrm{Fe}_{2} \mathrm{O}_{3} \mathrm{~T}$ contents, no systematic correlation seems to exist among opaque mineral size and abundance, total iron content, and magnetic properties.

The greater abundance of pillow breccias and hyaloclastites, the thicker and more common smectite with/without zeolites and/or calcium-carbonate veins, indicate that probably the uppermost section of the oceanic crust of Hole 896A was initially more permeable than the crust at Hole 504B. This fact has to be related to the setting of Site 896 on a basement topographic high, which has controlled localized hydrothermal upflow, and hence oceanic crust oxidizing alteration at low temperature. The latter, in turn, promoted secondary mineral precipitation as veins and as breccia and hyaloclastite cements. This situation appears similar to that at Hole 417A, at the foot of the Bermuda Rise, in the northwest Atlantic, where oxidizing low-temperature alteration of the oceanic crust prevailed along abundant thick secondary mineral veins and the breccias and hyaloclastites (Alt and Honnorez, 1984).

\section{ACKNOWLEDGMENTS}

We thank J.C. Alt, D.R. Peacor, and an anonymous reviewer for their critical reviews and insightful comments on a very preliminary version of our paper submitted prematurely to meet ODP deadline. We thank the crew and engineers who participated in Leg 148. This work was supported in part by the Geosciences Marines program of the Institut National des Sciences de l'Univers, France, and the Deutsche Forschungsgemeinschaft, Germany.

\section{REFERENCES}

Akimoto, T., Kinoshita, H., and Furuta, T., 1984. Electron probe microanalysis study on processes of low-temperature oxidation of titanomagnetite. Earth Planet. Sci. Lett., 71:263-278.

Alt, J.C., and Honnorez, J., 1984. Alteration of the upper oceanic crust, DSDP Site 417: mineralogy and chemistry. Contrib. Mineral. Petrol., $87: 149-169$.

Baksi, A.K., 1995. Fine tuning the radiometrically derived geomagnetic polarity time scale (GPTS) for 0-10 Ma. Geophys. Res. Lett., 22:457460 . 
Bleil, U., and Petersen, N., 1977. Magnetic properties of basement rocks, Leg 37, Site 332. In Aumento, F., Melson, W.G., et al., Init. Repts. DSDP. 37: Washington (U.S. Govt. Printing Office), 449-456.

Böhlke, J.K., Honnorez, J., Honnorez-Guerstein, B.-M., Muehlenbachs, K., and Petersen, N., 1981. Heterogeneous alteration of the upper oceanic crust: correlation of rock chemistry, magnetic properties, and $\mathrm{O}$ isotope ratios with alteration patterns in basalts from Site 396B, DSDP. J. Geophys. Res., 86:7935-7950.

Heinrich, E.W., 1965. Microscopic Identification of Minerals: New York (McGraw-Hill).

Hobart, M.A., Langseth, M.G., and Anderson, R.N., 1985. A geothermal and geophysical survey on the south flank of the Costa Rica Rift: Sites 504 and 505. In Anderson, R.N., Honnorez, J., Becker, K., et al., Init. Repts. DSDP, 83: Washington (U.S. Govt. Printing Office), 379-404.

Honnorez, J., 1972. La Palagonitisation: l'alteration sous-marine du verre volcanique basique de Palagonia (Sicile) [Palagonitization: the submarine alteration of basic volcanic glass in Palagonia, Sicily]. Publ. Vulkaninst. Immanuel Friedlaender, 9.

1981. The aging of the oceanic crust at low temperature. In Emiliani, C. (Ed.), The Sea (Vol. 7): The Oceanic Lithosphere: New York (Wiley), 525-587.

Honnorez, J., Böhlke, J.K., Honnorez-Guerstein, B.-M., and Petersen, N., 1978. Correlation between the changes in the mineralogy, chemistry, and magnetic properties of basalts from DSDP Leg 46 with low temperature submarine alteration. In Dmitriev, L., Heirtzler, J., et al., Init. Repts. DSDP, 46: Washington (U.S. Govt. Printing Office), 371-376.

Johnson, H.P., 1979. Magnetization of the oceanic crust. Rev. Geophys. Space Phys., 17:215-226.

Kelso, P.R., Banerjee, S.K., and Worm, H.-U., 1991. The effect of low-temperature hydrothermal alteration on the remanent magnetization of synthetic titanomagnetites: a case for acquisition of chemical remanent magnetization. J. Geophys. Res., 96:19545-19553.

Kent, D.V., and Gee, J., 1994. Grain size-dependent alteration and the magnetization of oceanic basalts. Science, 265:1561-1563.

Kronmüller, H., and Walz, F., 1980. Magnetic after-effect in magnetite and the vacancy doped magnetite. Philos. Mag. B, 42:433-452.

Langseth, M.G., Mottl, M.J., Hobart, M.A., and Fisher, A., 1988. The distribution of geothermal and geochemical gradients near Site 501/504: implications for hydrothermal circulation in the oceanic crust. In Becker, K., Sakai, H., et al., Proc. ODP, Init. Repts., 111: College Station, TX (Ocean Drilling Program), 23-32.

Laverne, C., and Vivier, G., 1983. Petrographical and chemical study of basement basalts from the Galapagos Spreading Center, Leg 70. In Honnorez, J., Von Herzen, R.P., et al., Init. Repts. DSDP. 70: Washington (U.S. Govt. Printing Office), 375-389.
Mottl, M.J., 1989. Hydrothermal convection, reaction, and diffusion in sediments on the Costa Rica Rift flank: pore-water evidence from ODP Sites 677 and 678. In Becker, K., Sakai, H., et al., Proc. ODP. Sci. Results, 111: College Station, TX (Ocean Drilling Program), 195-213.

Nishitani, T., and Kono, M., 1983. Curie temperature and lattice constant of oxidized titanomagnetite. Geophys. J. R. Astron. Soc., 74:585-600.

O'Donovan, J.B., and O'Reilly, W., 1983. Magnetic properties of basalts from Hole 504B, Deep Sea Drilling Project Leg 69. In Cann, J.R., Langseth, M.G., Honnorez, J., Von Herzen, R.P., White, S.M., et al., Init. Repts. DSDP, 69: Washington (U.S. Govt. Printing Office), 721-726.

O'Reilly, W., 1984. Rock and Mineral Magnetism: Glasgow (Blackie and Son).

Pariso, J.E., and Johnson, H.P., 1991. Alteration processes at Deep Sea Drilling Project/Ocean Drilling Program Hole 504B at the Costa Rica Rift: implications for magnetization of oceanic crust. J. Geophys. Res., 96:11703-11722.

Prévot, M., and Bina, M.M., 1993. Origin of magnetic viscosity and estimate of long-term induced magnetization in coarse grained submarine basalts. Geophys. Res. Lett., 20:2483-2486.

Prévot, M., Lecaille, A., and Mankinen, E.A., 1981. Magnetic effects of maghemitization of oceanic crust. J. Geophys. Res., 86:4009-4020.

Readman, P.W., and O'Reilly, W., 1972. Magnetic properties of oxidized (cation-deficient) titanomagnetites $(\mathrm{Fe}, \mathrm{Ti}, \mathrm{O})_{3} \mathrm{O}_{4}$. J. Geomagn. Geoelectr., 24:69-90.

Ryall, P.J.C., and Hall, J.M., 1980. Iron loss in titanomagnetites during low temperature oxidation. J. Geomagn. Geoelectr., 32:661-669.

Schmidbauer, E., and Fassbinder, J., 1987. After-effect of magnetic susceptibility in Fe-Ti spinels and cation diffusion. J. Magn. Magn. Mater., 68:83-89.

Seyfried, W.E., Jr., and Mottl, M.J., 1982. Hydrothermal alteration of basalt by seawater under seawater-dominated conditions. Geochim. Cosmochim. Acta, 46:985-1002.

Shipboard Scientific Party, 1993. Site 896. In Alt, J.C., Kinoshita, H., Stokking, L.B., et al., Proc. ODP, Init. Repts., 148: College Station, TX (Ocean Drilling Program), 123-192.

Stacey, F.D., and Banerjee, S.K., 1974. The Physical Principles of Rock Magnetism. Dev. Solid Earth Geophys., 5: New York (Elsevier).

Worm, H., and Banerjee, S.K., 1984. Aqueous low-temperature oxidation of titanomagnetite. Geophys. Res. Lett., 11:169-172.

Date of initial receipt: 19 August 1994

Date of acceptance: 10 August 1995

Ms 148SR-121 

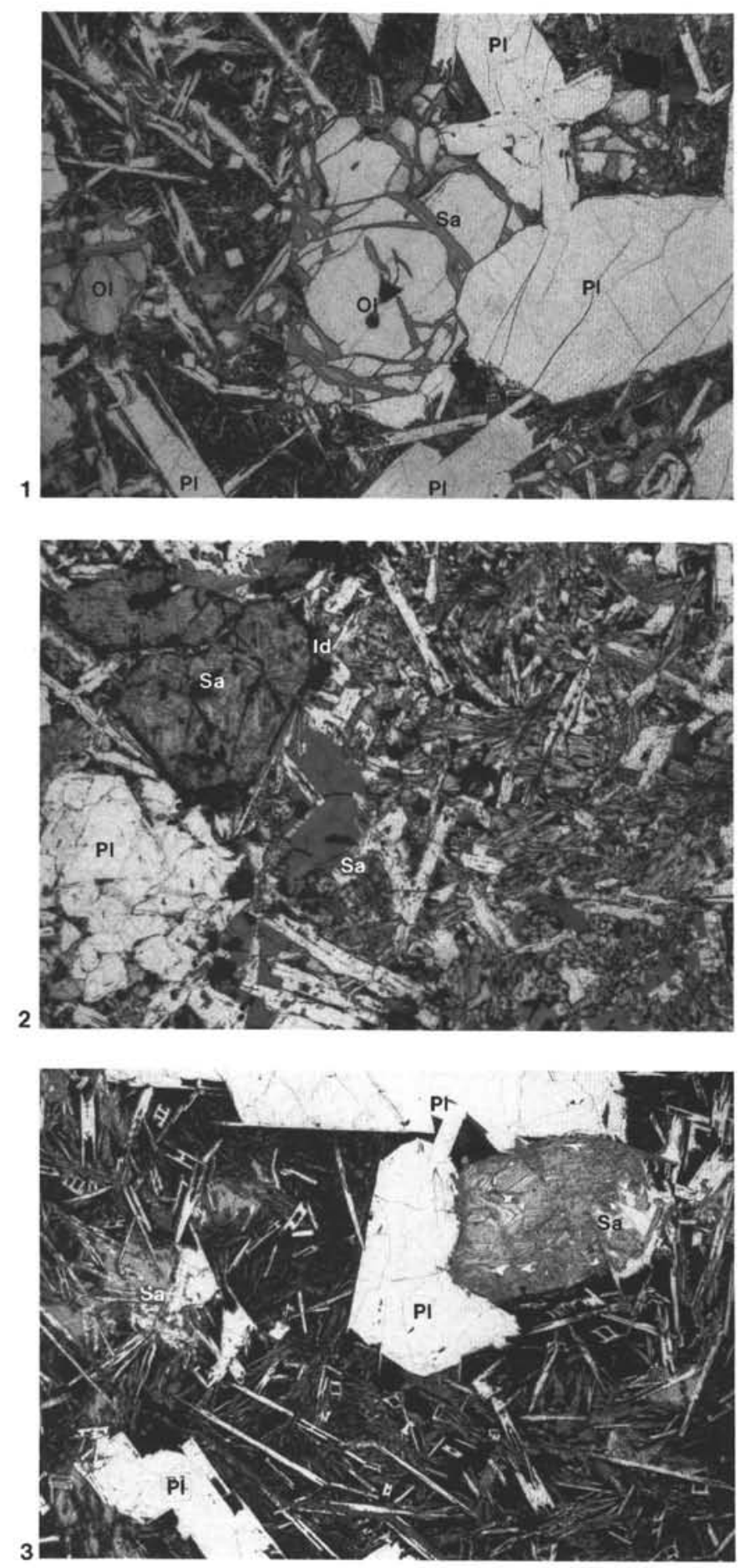

Plate 1. Photomicrographs in transmitted light, polars not crossed. 1. Sample 148-896A-11R-3, 89-91 cm (Piece 5), shows incipient replacement of olivine $(\mathrm{Ol})$ by saponite along cracks and around phenocryst outlines whereas plagioclase $(\mathrm{Pl})$ is unaltered. Interstitial voids are filled with, or glass is replaced by, homogeneous fine-grained saponite. Large unaltered spinel is in upper right corner. The olivine phenocryst in center with triangle-shaped inclusion is $1.8 \mathrm{~mm}$ long. 2. Sample 148-896A-16R-2, 21-26 cm (Piece 1C), coarsely crystallized saponite ( $\mathrm{Sm}$ ) pseudomorph after two olivine phenocrysts with iddingsite (Id) along crystal outlines and cracks. Notice that plagioclase phenocryst adjacent to olivine remains unaltered. Two vugs at center are filled with fined grained saponite. The larger of the two olivine phenocrysts is $1.2 \mathrm{~mm}$ long. 3. Sample 148-896A-6R-2, 67-70 cm (Piece 8), has a coarsely crystallized, fibrous saponite pseudomorph after an olivine phenocryst, whereas all the plagioclase phenocrysts and microlites are unaltered. Fine-grained, homogeneous saponite fills out interstitial miarolitic voids (or replaces glass?) A $0.06-\mathrm{mm}$-thick veinlet of saponite is barely visible in the lower left corner. 
Plate 2. Photomicrographs in transmitted light, polars not crossed. 1. Sample 148-896A-23R-2, 19-21 cm (Piece 2A), shows several coarsely crystallized saponite pseudomorphs after plagioclase phenocrysts. Notice the preferential replacement of phenocryst cores by the clay mineral with large relicts of primary plagioclase, whereas the albitic rims are unaltered. A small olivine phenocryst is totally replaced by saponite, with iddingsite along inner cracks and contours. A 0.15 -mm-thick saponite veinlet diagonally crosses the field, starting from the top right of the photograph, and a 0.3- to 0.5-mm-thick saponite vein lines the left side of the photograph. 2. Same sample as in Figure 4 but with a 0.15 - to 0.22 -mm-thick zoned saponite veinlet that crosses the field from left to right side. Notice replacement by saponite of the cores of plagioclase microphenocrysts and microlites near the veinlet, leaving unaltered albitic rims. 3. Sample 148-896A-21R-2, 117-119 cm (Piece 11B), contains a 0.3 -mm-thick vein of coarsely crystallized, pelletoidal saponite with fibroradial phillipsite $(\mathrm{Ph})$ prism aggregates near the selvages but not directly in contact with the host-rock walls of the vein. In the right lower corner are coarsely crystallized saponite pseudomorphs after three olivine phenocrysts; the largest one is $1 \mathrm{~mm}$ long. The cores of several plagioclase microphenocrysts (about $0.3-0.5 \mathrm{~mm}$ long) have been replaced by coarsely crystallized saponite, near the field lower center.
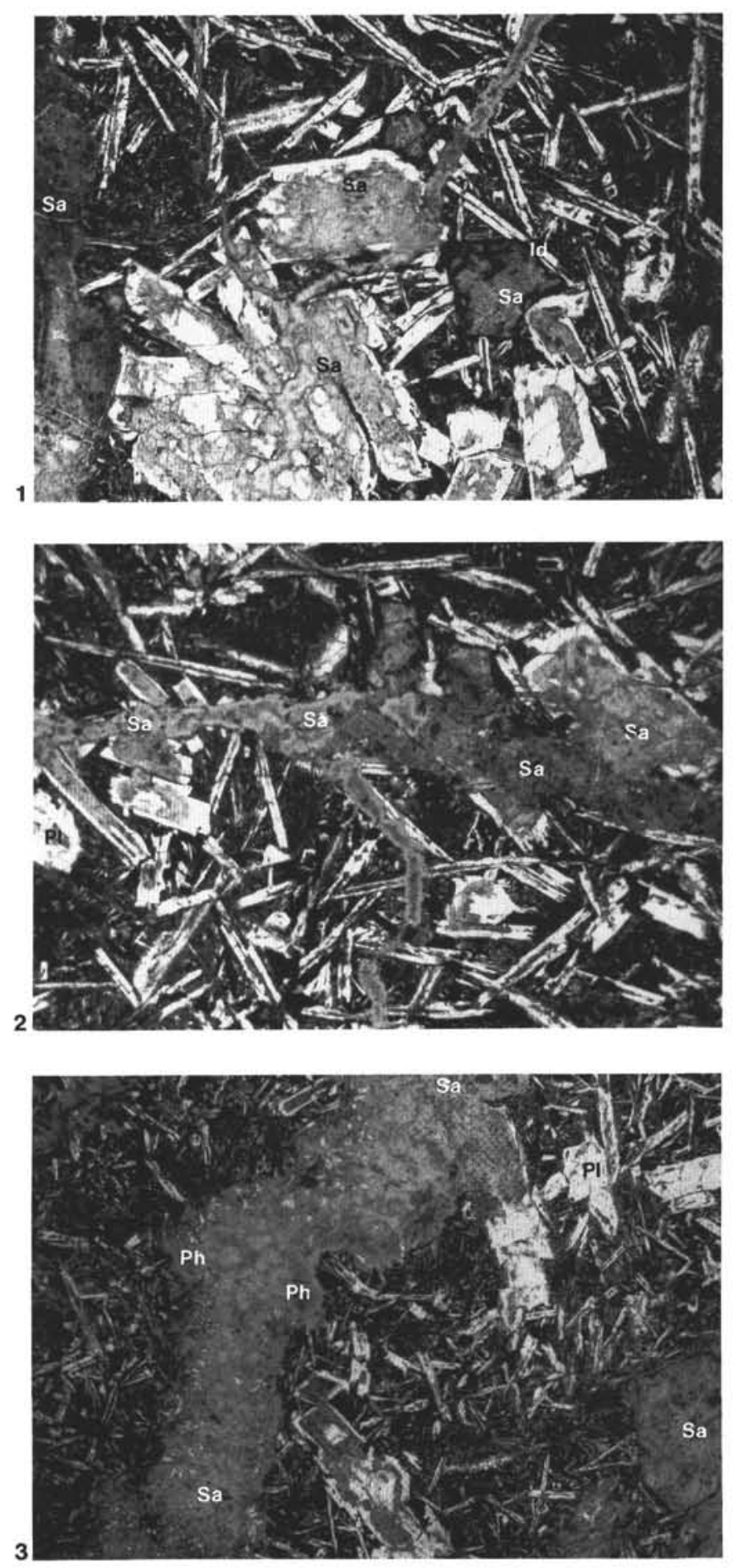

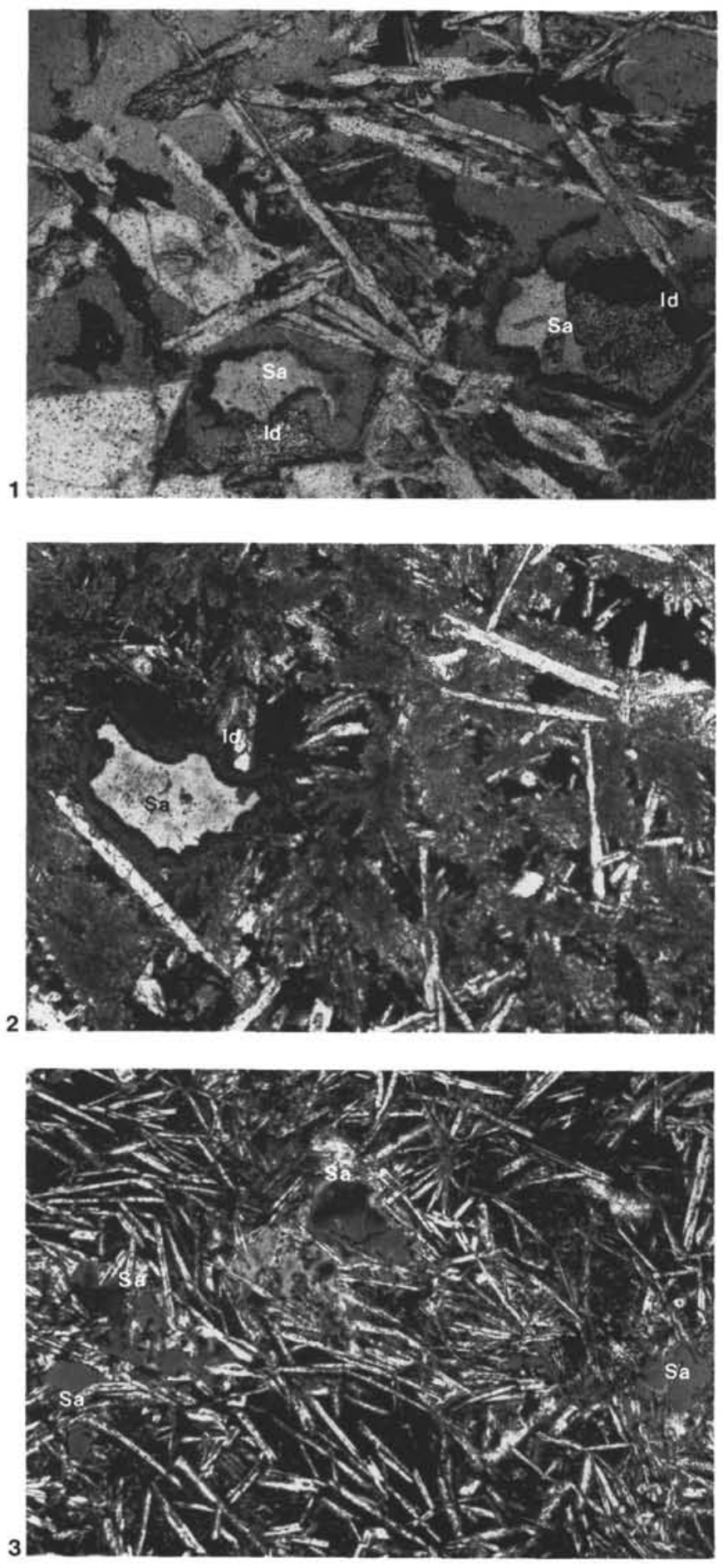

Plate 3. Photomicrographs in transmitted light, polars not crossed. 1. Sample 148-896A-1 IR-1, 50-52 cm (Piece 3E), has three miarolitic voids filled with concentrically zoned and locally botryoidal layers of reddish brown iddingsite near the walls and almost colorless, fibrous saponite at the center. The miarolitic voids in the lower left quarter are $0.3 \mathrm{~mm}$ long. 2 . Sample 148$896 \mathrm{~A}-17 \mathrm{R}-2,18-20 \mathrm{~cm}$ (Piece 3A), contains a 0.32 -mm-long vug filled with concentrically zoned botryoidal secondary minerals (dark reddish brown iddingsite along the vug walls and nearly colorless saponite at center. 3 . Sample 148-896A-12R-1, 52-54 cm (Piece 18), has a brown mottled color resulting from the fine-grained, rather homogeneous, orange-brown saponite filling miarolitic voids (or replacing interstitial glass?). Notice "desiccation cracks" filled with an unidentified Fe-oxyhydroxide in saponite-filled void (about $0.65 \mathrm{~mm}$ long) near the center. 
Plate 4. Photomicrographs in reflected light, polars not crossed. 1. Sample 148-896A-2R-1, 0-5 cm (Piece 1), has discrete, anhedral and/or partly skeletal granules of $\mathrm{Fe}$-Ti oxides ranging from $<1$ to $10 \mu \mathrm{m}$ in length. This sample displays a fine intergranular texture. 2. Sample 148-896A-IIR-1, 50-52 cm (Piece 3), has the same description as the sample in Figure 1, but it is shown at $2.5 \times$ higher magnification to illustrate the details of small skeletal $\mathrm{Fe}-\mathrm{Ti}$ oxide crystals up to $10 \mu \mathrm{m}$ in diameter. 3. Sample 148-896A-1 IR-3, 89-91 $\mathrm{cm}$ (Piece 15), is the second coarsest intergranular basalt, with Fe-Ti oxide either as skeletal crystals up to $50 \mu \mathrm{m}$ long or as rounded anhedral grains ranging from $<1$ to $20 \mu \mathrm{m}$ in diameter. Discrete drop-shaped sulfide granules are attached to $\mathrm{Fe}-\mathrm{Ti}$ oxide, $<2 \mu \mathrm{m}$ in diameter.
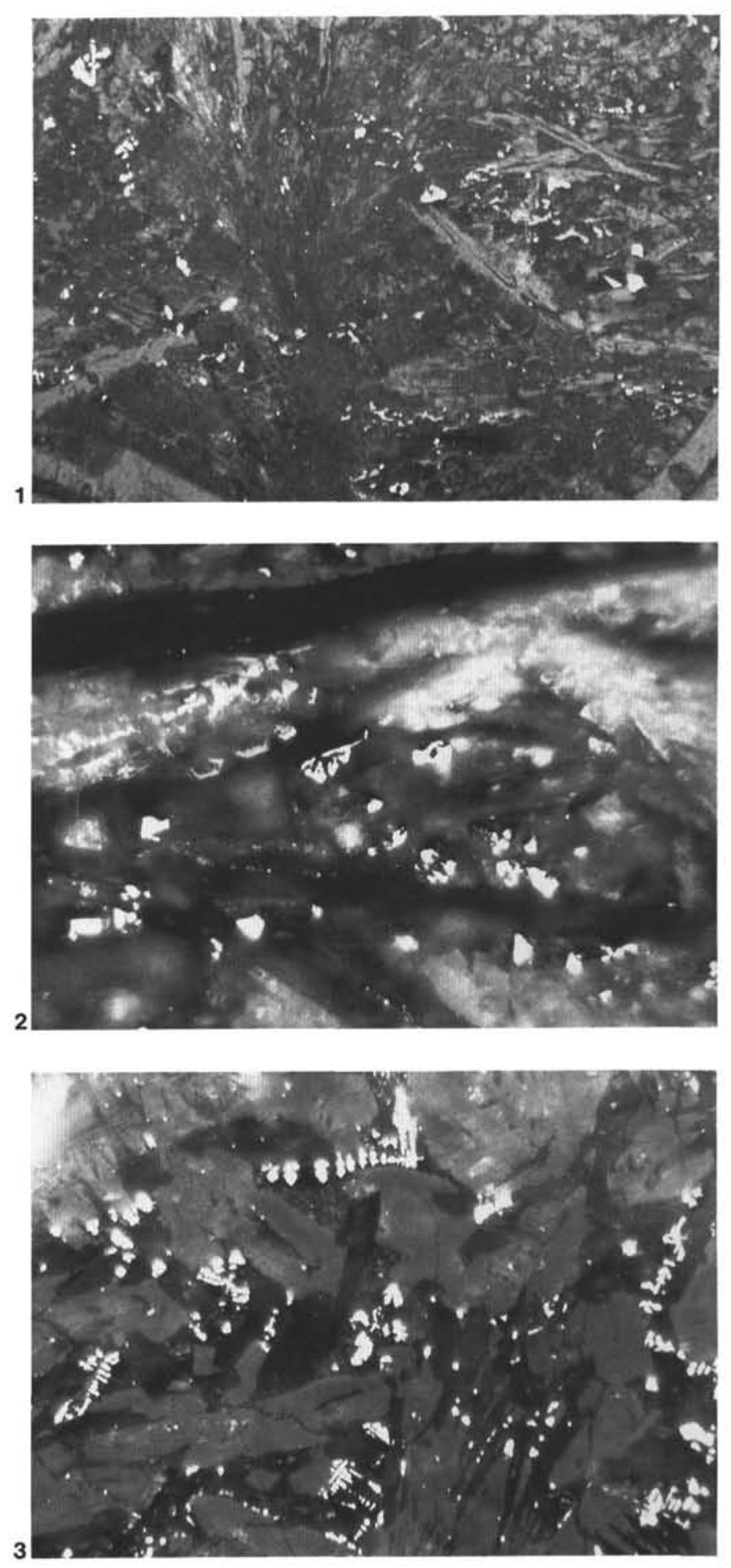

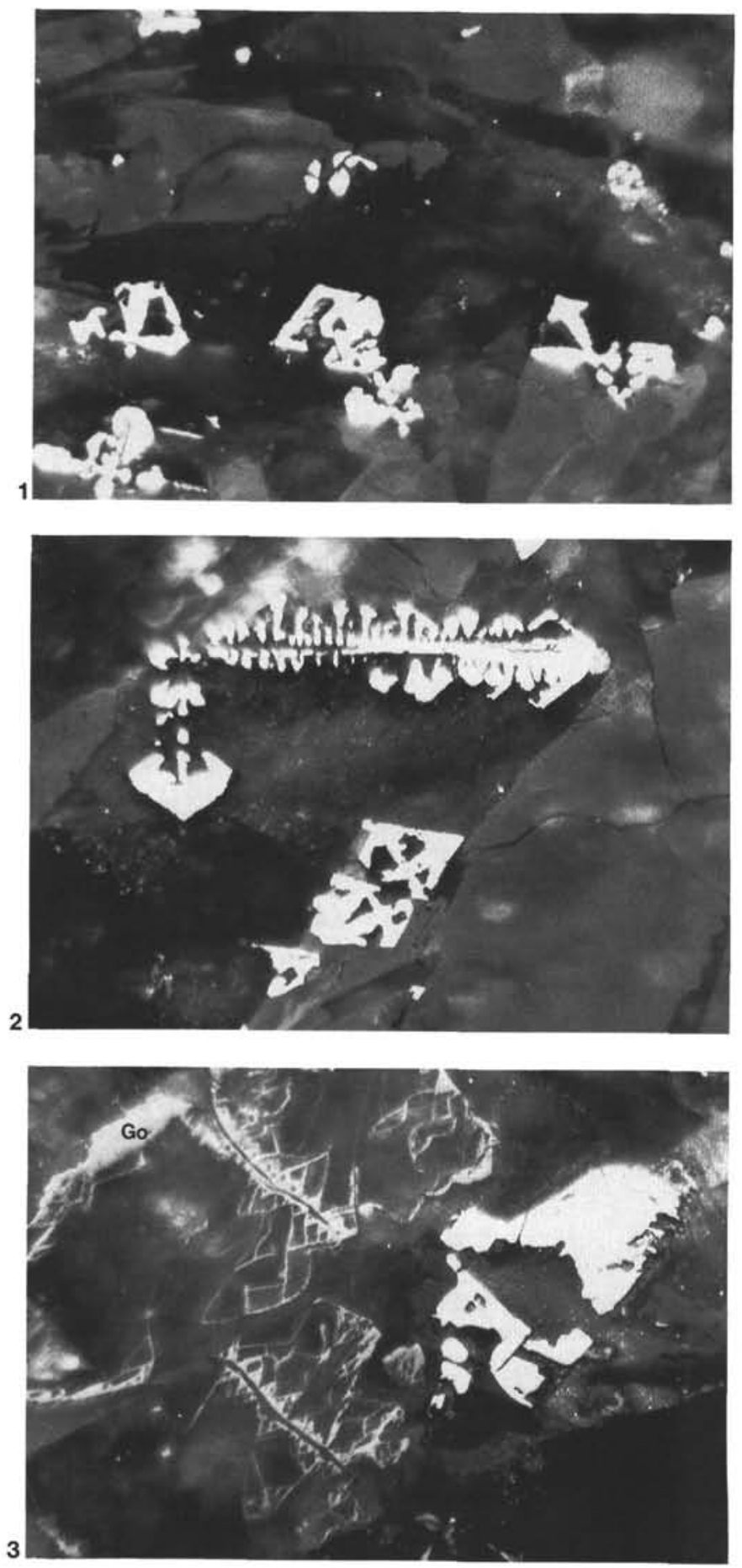

Plate 5. Photomicrographs in reflected light, polars not crossed. 1. Sample 148-896A-16R-3, 84-86 cm (Piece 4), shows Fe-Ti oxide in a coarse intergranular basalt, either as relatively large skeletal crystals up to $25 \mu \mathrm{m}$ long or as smaller, rounded, anhedral grains ranging from 1 to $<5 \mu \mathrm{m}$ in diameter. 2 . Same sample as in Figure 1, showing skeletal Fe-Ti oxide crystals, up to 200 $\mu \mathrm{m}$ long. The largest, horizontal Fe-Ti oxide crystal shows a drop-shaped oxidized sulfide granule to the right end of its growth axis. 3. Sample 148$896 \mathrm{~A}-16 \mathrm{R}-2,26-28 \mathrm{~cm}$ (Piece 1C), is the coarsest intergranular basalt, with discrete, anhedral crystals of Fe-Ti oxide (the largest one is $40 \mu \mathrm{m}$ long), lightning-like goethite (Go), and unidentified $\mathrm{Fe}$-oxyhydroxydes along cracks in two smectite pseudomorphs after olivine phenocrysts. 